\title{
Games on graphs
}

\author{
Benjamin Allen and Martin A. Nowak
}

\begin{abstract}
Evolution occurs in populations of reproducing individuals. The trajectories and outcomes of evolutionary processes depend on the structure of the population. Evolutionary graph theory is a powerful approach to studying the consequences of spatial or social population structure. The vertices of the graph represent individuals. The edges determine who interacts with whom for game payoff and who competes with whom for reproduction. Interaction and competition can be governed by the same graph or by two different graphs. In this paper, we review the basic approach for evolutionary games on graphs and provide new proofs for key results. We formalize the method of identity by descent to derive conditions for strategy selection on finite, weighted graphs. We generalize our results to nonzero mutation rates, and to the case where the interaction and competition graphs do not coincide. We conclude with a perspective of open problems and future directions.
\end{abstract}

Mathematics Subject Classification (2010). 91A22; 92B05, 60J20.

Keywords. Game theory, graph theory, evolution, Markov chain, cooperation.

\section{Introduction}

The mathematical study of evolution dates back to the work of Fisher, Haldane and Wright, who laid the foundations of population genetics in the first half of the 20th century. Mathematical modeling has become an indispensable tool in understanding the traits, behaviors, and patterns that arise from the fundamental processes of

B. Allen, Department of Mathematics, Emmanuel College, Boston, Massachusetts, U.S.A. and Program for Evolutionary Dynamics, Harvard University, Cambridge, Massachusetts, U.S.A.

E-mail: benjcallen@gmail.com

M. A. Nowak, Program for Evolutionary Dynamics, Harvard University, Cambridge, Massachusetts, U.S.A. and Department of Mathematics, Department of Organismic and Evolutionary Biology, Harvard University, Cambridge, Massachusetts, U.S.A.

E-mail: martin_nowak@harvard.edu 
mutation and natural selection. Precise mathematical descriptions of evolutionary dynamics tell us what to look for empirically and how to interpret our findings.

Much early work on the mathematics of evolution deals with constant selection, meaning that the reproductive rate of an individual is constant and depends only on its own genetic material. Evolutionary game theory [54], [53], [17], [89], [118], [37], [38], [66], [12] is the study of evolution when selection depends on the relative abundance (= frequency) of strategies or phenotypes in the population. Interactions that affect fitness are conceptualized as a game, with payoffs linked to reproductive success.

Among other successes, evolutionary game theory has helped elucidate one of the foundational questions of evolutionary theory: the problem of cooperation. From microbes to humans, organisms cooperate in a variety of ways, often reducing their own reproductive fitness to aid others. Mathematical analysis has shown that these forms of costly cooperation cannot evolve unless there is some mechanism in the evolutionary process that supports them [59].

Spatial structure is one such mechanism. Cooperators form clusters which can prevail in competition with defectors. An approach to spatial evolutionary game theory was formulated by Nowak and May [63], [64], who studied the Prisoners' Dilemma and other games on lattices. In their model, interactions occur between neighbors and successful individuals reproduce into neighboring sites. They found that, depending on the payoff matrix, either cooperators or defectors may take over the population, or they may coexist in either static or dynamic configurations. Their work inspired many further investigations of evolutionary game dynamics on lattices [62], [61], [22], [43], [57], [9], [55], [36], [81], [82].

More generally, spatial structure can be represented as a graph [48], [95]. Vertices represent individuals, and edges represent spatial or social connections between them. Individuals interact with neighbors on the graph, receiving an aggregate payoff. Births and deaths then occur according to some update rule [48], [68]. The update rule is an important aspect of the evolutionary process - different update rules can lead to different traits being selected.

For example, let us consider a simplified Prisoner's Dilemma on a regular graph of degree $k$. There are two types of strategies. Cooperators pay a cost, $c$, for their neighbors to receive a benefit, $b$. Defectors pay no cost and distribute no benefit. It turns out that the "death-birth" update rule, which will be explained below, favors evolution of cooperation if the benefit-to-cost ratio exceeds the graph degree, $b / c>k$ [68]. The fewer the number of neighbors per individual, the greater the scope for cooperation to evolve. In contrast, for "birth-death" updating, cooperation is never favored. These results were obtained with pair approximation [51], [21], a method that is known to be inexact in general (e.g. [92], [104]); however, they agree with later results obtained by exact methods [16], [14]. The $b / c>k$ rule has since been generalized to weighted graphs [110], [32] and to evolution with positive mutation rate [5]. 
The main goal of this work is to review and provide new proofs for the $b / c>k$ rule and its generalizations. We use the method of identity-by-descent [50], which has the advantage of being both mathematically elegant and biologically intuitive. A secondary goal is to develop a formalism allowing this method to be applied to evolutionary games on graphs. We focus primarily on exact results for graphs with symmetry, complementing earlier reviews [95], [82], [87] that take a wider view of the field.

Our review is organized as follows. In Section 2 we present the basic model for evolutionary games on graphs, and review a variety of update rules compatible with this model. Section 3 presents a mathematical formalism for the method of identity-by-descent. In Section 4 we apply this method to obtain conditions for the success of cooperation in a simplified Prisoner's Dilemma, including the $b / c>k$ rule and its generalization to arbitrary games on finite weighted graphs. Sections 5 and 6 , respectively, further generalize these results to evolution with nonzero mutation rates, and to populations whose interaction graph differs from the replacement graph. Finally, in Section 7, we briefly review further directions and open problems in evolutionary games on graphs. The proofs of intermediate lemmas are given in the appendices.

\section{Model: evolutionary games on graphs}

2.1. Graph representation of population structure. We consider a population structured as a weighted graph $G$ with $N \geq 2$ vertices. Each vertex represents an individual. The edge weight $e_{i j}$ represents the frequency of interaction and replacement between vertices $i, j \in G$. We suppose the edges are undirected, $e_{i j}=e_{j i}$, and normalized so that $\sum_{j \in G} e_{i j}=1$ for each $i \in G$. We also suppose that $G$ has vertex-transitive symmetry, so that for any pair of vertices $i, j \in G$, there is an isomorphism $\phi$ of $G$ such that $\phi(i)=j$. Intuitively, this symmetry condition means that the graph looks the same from the vantage point of any vertex.

We define the Simpson degree, $\kappa$, of a graph as [2]

$$
\kappa=\left(\sum_{j \in G} e_{i j}^{2}\right)^{-1} .
$$

The Simpson degree can be understood as the Simpson diversity [88] of neighbors per vertex. It is equal to the inverse likelihood that two randomly chosen neighbors (chosen with replacement and with probability proportional to edge weight) are the same. The special case of an unweighted graph is recovered by supposing that all edges have weight $1 / k$, where $k$ is the graph degree. In this case we obtain $\kappa=k$. Thus, the Simpson degree generalizes the usual notion of graph degree (Figure 1).

In general, the graph $G$ can have self-loops, which means that the diagonal values, $e_{i i}$, can be positive. Self-loops allow an individual to interact with itself via 


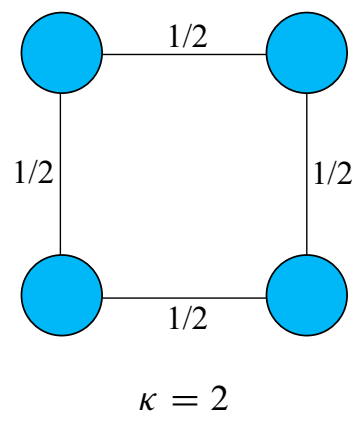

(a)

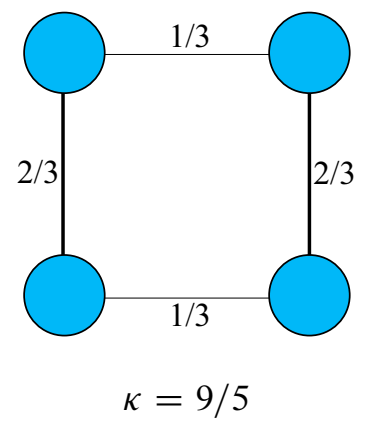

(b)

Figure 1. The Simpson degree $\kappa=\left(\sum_{j \in G} e_{i j}^{2}\right)^{-1}$ for two weighted transitive graphs. Note that for graph (a), which has equal edge weights to each neighbor, the Simpson degree equals the graph degree.

the game and/or replace itself. In the case of no mutation (see Section 2.4), selfreplacement leaves the population state unchanged and the only effect of a self-loop is self-interaction. If there is mutation, then self-replacement can change the state of the population.

2.2. Game interaction and payoff. Interactions are modeled as a symmetric $2 \times 2$ game with payoff matrix

$$
\begin{gathered}
\mathrm{C} \\
\mathrm{C} \\
\mathrm{D}
\end{gathered}\left(\begin{array}{ll}
R & S \\
T & P
\end{array}\right)
$$

While the methods used here apply to arbitrary $2 \times 2$ payoff matrices, we use a notation that is suggestive of cooperative dilemmas [60], with $\mathrm{C}$ representing cooperation and $\mathrm{D}$ representing defection. In this context, $R$ denotes the reward for a pair of cooperators, $T$ denotes the temptation to defect, $S$ denotes the "sucker" payoff for a cooperator being exploited by a defector, and $P$ denotes the punishment for a defecting pair. The most stringent form of cooperative dilemma is the Prisoners' Dilemma, defined by $T>R>P>S$.

At each time-step, each individual $i \in G$ retains the weighted average payoff

$$
f_{i}=\sum_{j \in G} e_{i j} g_{i j}
$$

where $g_{i j}$ is the payoff that $i$ receives from playing with $j$.

The payoffs $f_{i}$ are then transformed by a mapping

$$
f_{i} \mapsto F_{i}=F\left(\delta f_{i}\right),
$$


where $F(x)$ is a positive, increasing, differentiable function with $F(0)=F^{\prime}(0)=1$. For example, we can choose $F(x)=e^{x}$. The rescaled payoff $F_{i}$ represents reproductive rate or survival probability in models of genetic evolution, or the likelihood to be imitated in models of cultural evolution. The parameter $\delta \geq 0$ represents the strength of selection - i.e., the extent to which the game payoff affects reproductive success. We focus on weak selection (small positive $\delta$ ), in which case we have the expansion $F_{i}=1+\delta f_{i}+\mathcal{O}\left(\delta^{2}\right)$.

2.3. Update rules. To define evolutionary dynamics on graphs, one must choose an update rule - a scheme for determining birth and death events based on rescaled payoff. A variety of update rules have been considered, including those listed below (see also Figure 2). For consistency we describe these update rules in biological language; however, they can be instantiated by either genetic reproduction or social imitation.

- Birth-Death (BD) [68]: An individual $i \in G$ is chosen at random, with probability proportional to $F_{i}$, to reproduce. The offspring replaces the occupant of a neighboring vertex $j \in G$, chosen at random with probability proportional to $e_{i j}$.

- Death-Birth ( $D B)$ [68]: An individual $j \in G$ is chosen at random, with uniform probability, to be replaced. A neighboring individual $i \in G$ is chosen to reproduce, with probability proportional to $F_{i} e_{i j}$. The offspring of $i$ replaces the occupant of $j$.

- Pairwise comparison (PC) [96], [36], [84]: An individual $j \in G$ is chosen at random, with uniform probability, to potentially be replaced. A neighboring individual $i \in G$ is chosen with probability $e_{i j}$ to potentially reproduce. With probability $\theta\left(F_{i}-F_{j}\right)$, individual $i$ produces an offspring that replaces the occupant of $j$; otherwise, no reproduction occurs. Here $\theta$ is an increasing function that is bounded between 0 and 1 and has the property that $\theta(x)-\theta(-x)$ is differentiable at $x=0$. Examples include $\theta(x)=\left(1+e^{-x}\right)^{-1}$ [96], [36], [113] and $\theta(x)=x_{+} / M$, where $x_{+}=\max (x, 0)$ and $M$ is the maximum possible value of $F_{j}-F_{i}$ [36], [84].

- Imitation (IM) [68]: This update rule is defined only for unweighted graphs. An individual $j \in G$ is chosen, at random with uniform probability, to be replaced. An individual $i \in G$ is chosen from among $j$ and its immediate neighbors, with probability proportional to $F_{i}$, to reproduce. The offspring of $i$ replaces the occupant of $j$. Note that for IM updating, an individual can be replaced by its own offspring.

- Birth-death with payoff affecting death (BD-D) [32], [109]: An individual $i \in G$ is chosen at random, with uniform probability, to reproduce. The offspring replaces the occupant of a neighboring vertex $j \in G$, chosen at random with probability proportional to $e_{i j} / F_{j}$. 
- Death-birth with payoff affecting death (DB-D) [32], [109]: An individual $j \in G$ is chosen at random, with probability inversely proportional to $F_{j}$, to be replaced. A neighboring individual $i \in G$ is chosen to reproduce, with probability proportional to $e_{i j}$. The offspring of $i$ replaces the occupant of $j$.

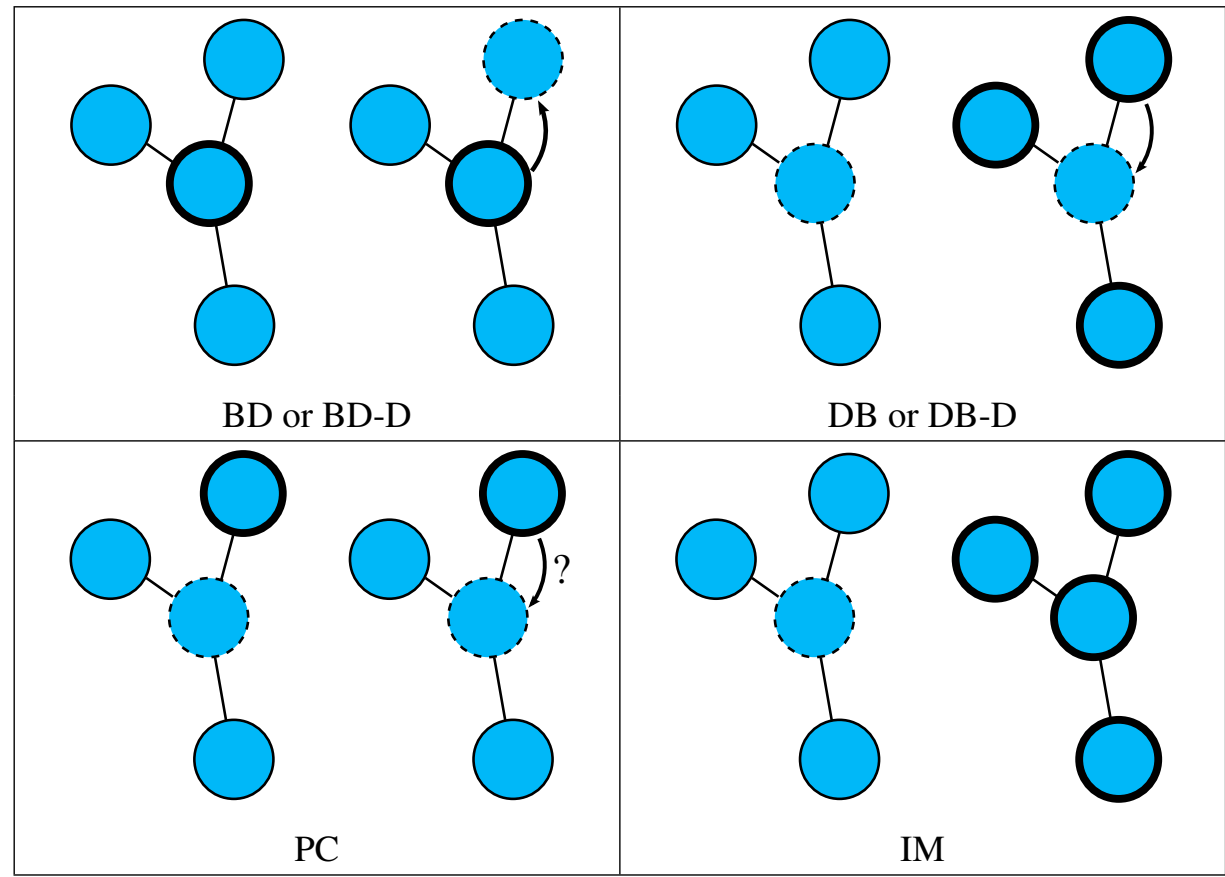

Figure 2. Schematic illustration of update rules on graphs. For BD, a reproducer is chosen proportionally to rescaled payoff, and its offspring replaces a neighbor chosen proportionally to edge weight. BD-D is the same as BD except that the reproducer is chosen uniformly and the neighbor to be replaced is chosen proportionally to (edge weight)/(rescaled payoff). For DB, a vertex is chosen uniformly to be replaced, and a neighbor is chosen proportionally to (edge weight $) \times($ rescaled payoff) to reproduce into this vertex. DB-D is the same as DB except that the vertex to be replaced is chosen inversely proportionally to rescaled payoff and the reproducing neighbor is chosen proportionally to edge weight. For PC, a vertex is chosen uniformly to potentially be replaced, and a neighbor is chosen proportionally to edge weight; this neighbor reproduces with probability depending on the difference in rescaled payoffs. For IM, a vertex is chosen uniformly to be replaced, and a reproducer is chosen proportionally to rescaled payoff from among this vertex and its neighbors. Formal descriptions are given in the main text.

2.4. Mutation. The mutation rate is denoted by $u$, where $0 \leq u \leq 1$. With probability $1-u$, the offspring inherits the type of the parent. With probability $u$, the offspring adopts type $\mathrm{C}$ or $\mathrm{D}$ with equal probability. 
2.5. The evolutionary Markov chain. Overall, this stochastic process is described by the evolutionary Markov chain $\mathcal{M}$. Each state can be written as a string $\boldsymbol{s}=$ $\left(s_{1}, \ldots, s_{N}\right) \in\{\mathrm{C}, \mathrm{D}\}^{N}$, where $s_{i} \in\{\mathrm{C}, \mathrm{D}\}$ denotes the type currently occupying vertex $i$. Summarizing the process described above, transitions in $\mathcal{M}$ occur as follows:

(1) Each individual $i \in G$ is assigned the rescaled payoff $F_{i}=F\left(\delta f_{i}\right)$, where $f_{i}$ is the weighted average payoff obtained from neighbors as given in Eq. (2).

(2) A reproducer $i \in G$ and an offspring location $j \in G$ are chosen according to the specified update rule.

(3) The new state $s^{\prime}=\left(s_{1}^{\prime}, \ldots, s_{N}^{\prime}\right)$ is determined by $s_{k}^{\prime}=s_{k}$ for all $k \neq j$ and

$$
s_{j}^{\prime}= \begin{cases}s_{i} & \text { with probability } 1-u, \\ \mathrm{C} & \text { with probability } u / 2, \\ \mathrm{D} & \text { with probability } u / 2\end{cases}
$$

\section{The method of identity by descent}

We study the assortment of types under this process using the idea of identity by descent (IBD) [50]. In biological terms, we say two individuals are IBD if no mutation separates either of them from their common ancestor. In particular, every individual is IBD to itself, and a child is IBD to its parent if and only if the child was born without mutation. At any given time IBD is an equivalence relation on the set of living individuals, whose equivalence classes can be understood as clonal subtypes of the population (Figure 3). Identity-by-descent methods have been applied to a variety of problems in evolution [91], [83], [110], [6], [116]. Here we present a formalism for identity-by-descent that enables its application to evolutionary games on graphs.

We introduce the IBD-enriched evolutionary Markov chain $\tilde{\mathcal{M}}$, an augmentation of $\mathcal{M}$ that incorporates the current IBD relation as an aspect of the state. States of $\widetilde{\mathcal{M}}$ are written as pairs $(\boldsymbol{s}, \iota)$, where $s \in\{\mathrm{C}, \mathrm{D}\}^{N}$ is a string describing the type occupying each vertex as before, and $\iota$ is an equivalence relation on the vertices of $G$. The IBD status of vertices $i, j \in G$ in a given state is described by the variable $\iota_{i j}$, which equals one if $i$ and $j$ are IBD and zero otherwise.

Transitions in $\widetilde{\mathcal{M}}$ occur by the following process: First, a reproducer $i \in G$ and an offspring location $j \in G$ are chosen according to Steps 1 and 2 of Section 2.5. Then the new state $\left(\boldsymbol{s}^{\prime}, \iota^{\prime}\right)$ is determined as follows:

- $s_{k}^{\prime}=s_{k}$ for all $k \in G \backslash\{j\}$. (The types of individuals who are not replaced remain unchanged.)

- $\iota_{k \ell}^{\prime}=\iota_{k \ell}$ for all $k, \ell \in G \backslash\{j\}$. (IBD relationships among individuals who are not replaced remain unchanged.) 


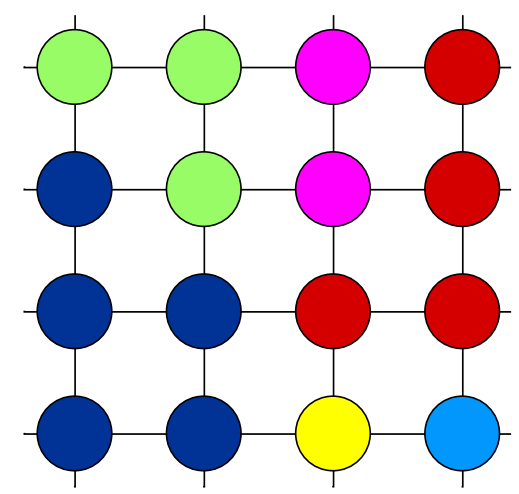

Figure 3. A sample identity-by-descent (IBD) partition on a lattice. Two individuals are IBD if no mutation separates either from their common ancestor. IBD is an equivalence relation on the set of living individuals, the equivalence classes of which represent clonal subtypes. In the IBD-enriched evolutionary Markov chain $\widetilde{\mathcal{M}}$, the current IBD partition is regarded as an aspect of the population state.

- $\iota_{j j}^{\prime}=1$. (The new individual is IBD to itself.)

- One of the following events occurs:

- with probability $1-u, s_{j}^{\prime}=s_{i}$ and $\iota_{j k}^{\prime}=\iota_{k j}^{\prime}=\iota_{k i}$ for all $k \neq j$,

- with probability $u / 2, s_{j}^{\prime}=\mathrm{C}$ and $\iota_{j k}^{\prime}=\iota_{k j}^{\prime}=0$ for all $k \neq j$,

- with probability $u / 2, s_{j}^{\prime}=\mathrm{D}$ and $\iota_{j k}^{\prime}=\iota_{k j}^{\prime}=0$ for all $k \neq j$.

(The first of these events represents faithful reproduction, in which case the new individual inherits the type and IBD relationships of its parent. The latter two represent mutation, in which case the new individual is equally likely to be either type and is IBD to none but itself.)

We denote the transition probability from state $(s, \iota)$ to state $\left(\boldsymbol{s}^{\prime}, \iota^{\prime}\right)$ in $\tilde{\mathcal{M}}$ by

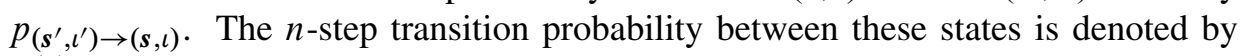
$p_{\left(s^{\prime}, l^{\prime}\right) \rightarrow(s, l)}^{(n)}$.

We observe that, in the IBD-enriched evolutionary Markov chain $\widetilde{\mathcal{M}}, s(t+1)$ is conditionally independent of $\iota(t)$, given $s(t)$. That is, changes in the distribution of types depend only on the current distribution of types, with no additional dependence on IBD relationships. Thus the evolutionary Markov chain $\mathcal{M}$ can be recovered from $\widetilde{\mathcal{M}}$ by forgetting the IBD component of the state.

The following lemma shows that, with mutation, $\tilde{\mathcal{M}}$ has a unique stationary distribution, under which individuals that are IBD are guaranteed to have the same type: 
Lemma 1. For $0<u \leq 1, \tilde{\mathcal{M}}$ has a unique stationary distribution $\left\{\pi_{(s, l)}\right\}(s, l)$. For any states $(\boldsymbol{s}, \iota)$ and $\left(\boldsymbol{s}^{\prime}, \iota^{\prime}\right), \lim _{n \rightarrow \infty} p_{\left(\boldsymbol{s}^{\prime}, \iota^{\prime}\right) \rightarrow(\boldsymbol{s}, l)}^{(n)}=\pi_{(\boldsymbol{s}, \iota)}$. Any state $(\boldsymbol{s}, \iota)$ with $\pi_{(s, l)}>0$ has the property that $s_{i}=s_{j}$ for any pair $i, j \in G$ with $\iota_{i j}=1$.

We denote expectations in the stationary distribution $\left\{\pi_{(s, l)}\right\}$ of $\tilde{\mathcal{M}}$ by using the brackets \langle\rangle .

3.1. Neutral drift. We now consider the case $\delta=0$, representing purely neutral drift between types $\mathrm{C}$ and $\mathrm{D}$. In this case, all of the update rules considered above reduce to the same process, which is also called the noisy voter model [33].

For neutral drift, the IBD relationships at time $t+1$ depend only on the IBD relationships at time $t$, in the sense that $\iota(t+1)$ is conditionally independent of $s(t)$ given $\iota(t)$. Specifically, for $i \neq j$, we have

$$
\iota_{i j}(t+1)= \begin{cases}\iota_{i j}(t) & \text { with probability }(N-2) / N, \\ \iota_{i \ell}(t) & \text { with probability } e_{\ell j}(1-u) / N \text { for each } \ell \in G, \\ \iota_{\ell j}(t) & \text { with probability } e_{\ell i}(1-u) / N \text { for each } \ell \in G, \\ 0 & \text { with probability } 2 u / N .\end{cases}
$$

3.2. IBD probabilities. We define the IBD probability $q_{i j}$ of vertices $i, j \in G$ to be the stationary probability that the occupants of these vertices are IBD under neutral drift: $q_{i j} \equiv\left\langle\iota_{i j}\right\rangle_{\delta=0}$. These IBD probabilities characterize the patterns of spatial assortment generated by neutral drift. Summing over all cases in Eq. (3), with $\iota_{i j}(t+1)$ and $\iota_{i j}(t)$ replaced by their stationary expectations $q_{i j}$, yields

$$
q_{i j}= \begin{cases}1, & i=j, \\ \frac{1-u}{2} \sum_{k \in G}\left(e_{k i} q_{k j}+e_{k j} q_{k i}\right), & i \neq j .\end{cases}
$$

To quantify how IBD probability scales with the distance between nodes, we define $q^{(n)}$ as the average IBD probability among nodes that are separated by a random walk of length $n$ in $G$ :

$$
q^{(n)}=\sum_{j \in G} p_{i j}^{(n)} q_{i j}=\frac{1}{N} \sum_{i, j \in G} p_{i j}^{(n)} q_{i j} .
$$

Above, $p_{i j}^{(n)}$ denotes the probability that a random walk of length $n$ starting at $i$ (with step probabilities given by edge weights) will terminate at $j$. Since edges are undirected, these probabilities are symmetric: $p_{i j}^{(n)}=p_{j i}^{(n)}$ for each $i, j \in G$. We use $p^{(n)}$ as shorthand for the probability $p_{i i}^{(n)}$ that such a walk terminates at its initial vertex. (By symmetry, this probability does not depend on the initial vertex $i$.) The probabilities $p^{(n)}$ and $q^{(n)}$ are related by the following lemma: 
Lemma 2. For all $0<u \leq 1$ and $n \geq 0$,

$$
q^{(n)}=p^{(n)}+\left(q^{(n+1)}-p^{(n)} q^{(1)}\right)(1-u) .
$$

Intuitively, Eq. (6) decomposes the probability $q^{(n)}$ - that individuals at two ends of an $n$-step random walk are IBD - into two cases: either they are the same individual (with probability $p^{(n)}$ ), or they are distinct and the younger one is the non-mutant offspring of a parent IBD to the older one. In the second case, the walk from the older one to the parent of the younger is $n+1$ steps long and does not return to its starting vertex at the $n$th step.

We now let $n \rightarrow \infty$ in Eq. (6). Vertex transitivity implies that $\lim _{n \rightarrow \infty} p_{i j}^{(n)}=$ $1 / N$ for all $i, j \in G$; that is, random walks become equally likely to terminate at each node as the walk length tends to infinity. Thus $p^{(n)}$ converges to $1 / N$ in this limit, while $q^{(n)}$ converges to $\bar{q}$, the probability that two individuals chosen randomly (with replacement) are IBD. Substituting in (6), we obtain

$$
(1-u) q^{(1)}=1-N u \bar{q} .
$$

Now substituting in Eq. (6) yields

$$
q^{(n)}=(1-u) q^{(n+1)}+N u \bar{q} p^{(n)} .
$$

Next we consider the limit of small mutation rate. From Eq. (4) we see that $\lim _{u \rightarrow 0} q_{i j}=1$ for all $i, j \in G$. (Intuitively, all individuals become IBD in the limit of rare mutation.) This observation implies the following low-mutation expansion of Eq. (8):

$$
q^{(n)}-q^{(n+1)}=u\left(N p^{(n)}-1\right)+O\left(u^{2}\right) .
$$

3.3. Assortment relative to a focal individual. We now consider the assortment of types around a typical individual of type $\mathrm{C}$. By symmetry, we may choose an arbitrary vertex $0 \in G$ as the focus of our analysis. We consider the stationary distribution on $\widetilde{\mathcal{M}}$ conditioned on 0 being $\mathrm{C}$. We denote expectations in this conditional distribution by \langle\rangle$_{\substack{\delta=0 \\ s_{0}=C}}$. The following lemma describes assortment under this conditional distribution:

Lemma 3. For all $0<u \leq 1$ and $i \in G$,
(i) $\operatorname{Pr}_{\delta=0}\left[s_{i}=\mathrm{C} \mid s_{0}=\mathrm{C}, \iota_{0 i}=1\right]=1$,
(ii) $\operatorname{Pr}_{\delta=0}\left[s_{i}=\mathrm{C} \mid s_{0}=\mathrm{C}, \iota_{0 i}=0\right]=\frac{1}{2}$,
(iii) $\operatorname{Pr}_{\delta=0}\left[s_{i}=\mathrm{C} \mid s_{0}=\mathrm{C}\right]=\frac{1+q_{0 i}}{2}$. 
In words, if it is given that vertex 0 has type $C$, then under neutral drift, (i) individuals IBD to 0 also have type $C$, (ii) individuals not IBD to 0 have $\frac{1}{2}$ probability to be $\mathrm{C}$ on average, and therefore (iii) overall, vertex $i$ has type $\mathrm{C}$ with probability $\left(1+q_{0 i}\right) / 2$.

To quantify how assortment scales with distance, we let $s^{(n)}$ denote the probability that the individual at the end of an $n$-step random walk from 0 is of type $\mathrm{C}$ in this conditional distribution:

$$
s^{(n)}=\sum_{i \in G} p_{0 i}^{(n)} \operatorname{Pr}_{\delta=0}\left[s_{i}=\mathrm{C} \mid s_{0}=\mathrm{C}\right]=\frac{1+q^{(n)}}{2} .
$$

\section{Conditions for evolutionary game success}

Using the above analysis of IBD probabilities, we now derive conditions for strategy selection under weak selection. We start with a simplified Prisoner's Dilemma game and then generalize to arbitrary games of the form (1).

4.1. Conditions for evolutionary success. We quantify evolutionary success in two ways, depending on whether mutation is present. If there is no mutation $(u=0)$, a natural metric for success is the fixation probability $\rho_{\mathrm{C}}$, defined as the probability of absorption in the all-C state $\mathbf{C}=(\mathrm{C}, \ldots, \mathrm{C})$ of $\mathcal{M}$, from an initial state with one $\mathrm{C}$ and the rest $\mathrm{D}$. The reverse fixation probability $\rho_{\mathrm{D}}$ is defined as the probability of absorption in $\mathbf{D}=(\mathrm{D}, \ldots, \mathrm{D})$, from an initial state with one $\mathrm{D}$ and the rest $\mathrm{C}$. We say that cooperation is favored if $\rho_{\mathrm{C}}>\rho_{\mathrm{D}}$.

If there is mutation $(u>0)$, we instead consider the expected frequency $\langle x\rangle$ of cooperators under the stationary distribution, where $x$ denotes their frequency in a particular state:

$$
x=\frac{1}{N}\left|\left\{i \in G: s_{i}=\mathrm{C}\right\}\right|
$$

We say cooperation is favored if cooperators are more abundant than defectors on average - that is, if $\langle x\rangle>\frac{1}{2}$.

In the low-mutation limit $(u \rightarrow 0)$, the conditions $\rho_{\mathrm{C}}>\rho_{\mathrm{D}}$ and $\langle x\rangle>\frac{1}{2}$ coincide with each other [4]. In fact, they both become equivalent to the condition [67]

$$
\left\langle\frac{\partial\left(b_{0}-d_{0}\right)}{\partial \delta}\right\rangle_{\substack{\delta=0 \\ s_{0}=\mathrm{C}}}>0 .
$$

Above, 0 denotes the focal individual, $b_{0}$ is the probability that this individual reproduces in a given state, and $d_{0}$ is the probability that it is replaced. The brackets \langle\rangle$_{\substack{\delta=0 \\ s_{0}=\mathrm{C}}}$ denote expectations in the stationary distribution of the neutral drift process, conditioned on 0 being of type $\mathrm{C}$. The relations among these success conditions are made precise in the following theorem: 
Theorem 4. Consider the family of evolutionary Markov chains $\mathcal{M}_{u, \delta}$ with fixed game, graph, and update rule, where the mutation rate $0 \leq u \leq 1$ and selection strength $\delta>0$ are allowed to vary. Then

(1) Strategy $\mathrm{C}$ is favored for fixed $u>0$, in the sense that $\langle x\rangle_{u, \delta}>1 / 2$ for all sufficiently small $\delta>0$, if and only if

$$
\left\langle\frac{\partial\left((1-u) b_{0}-d_{0}\right)}{\partial \delta}\right\rangle_{\substack{\delta=0 \\ s_{0}=\mathrm{C}}}>0 .
$$

(2) Strategy $\mathrm{C}$ is favored in the sense that $\langle x\rangle_{u, \delta}>1 / 2$ for all sufficiently small $u>0$ and $\delta>0$ if and only if

$$
\lim _{u \rightarrow 0}\left\langle\frac{\partial\left(b_{0}-d_{0}\right)}{\partial \delta}\right\rangle_{\substack{\delta=0 \\ s_{0}=\mathrm{C}}}>0 .
$$

(3) Strategy $\mathrm{C}$ is favored for $u=0$, in the sense that $\rho_{\mathrm{C}}>\rho_{\mathrm{D}}$ for all sufficiently small $\delta>0$, if and only if

$$
\lim _{u \rightarrow 0}\left\langle\frac{\partial\left(b_{0}-d_{0}\right)}{\partial \delta}\right\rangle_{\substack{\delta=0 \\ s_{0}=\mathrm{C}}}>0 .
$$

The factor $1-u$ in Condition (12) represents a mutational bias due to differential birth rates; for example, if type $\mathrm{C}$ reproduces more often than type $\mathrm{D}$, there will be more mutations from $\mathrm{C}$ to $\mathrm{D}$ than vice versa. This effect disappears in the limit of low mutation, or if either birth or death rates are constant across individuals in every state [67].

4.2. The simplified Prisoner's Dilemma. We first consider a simplified Prisoners' Dilemma game in which cooperators pay a cost $c>0$ to generate a benefit $b>c$ to the other player. The payoff matrix for this simplified game is

$$
\begin{aligned}
& \text { C D } \\
& \text { C } \left.\begin{array}{cc}
b-c & -c \\
b & 0
\end{array}\right) \text {. }
\end{aligned}
$$

We let $f^{(n)}$ denote the expected payoff of an individual at the terminus of an $n$-step walk from the focal individual 0 , conditioned on 0 being of type C. From the game matrix (13), we see that

$$
f^{(n)}=-c s^{(n)}+b s^{(n+1)}=\frac{1}{2}\left(-c+b-c q^{(n)}+b q^{(n+1)}\right) .
$$

We now separate into cases based on the update rule. 
4.2.1. Birth-Death, pairwise comparison, and DB-D updating. For BD, PC, or DB-D updating, Condition (11) reduces to $f^{(0)}>f^{(1)}$. That is, cooperation is favored if and only if the focal cooperator has greater payoff than its neighbors, on average. This result follows directly from the definitions of these update rules and the assumed properties of the rescaling function $F$.

By Eq. (14), the condition $f^{(0)}>f^{(1)}$ is equivalent to

$$
-c\left(q^{(0)}-q^{(1)}\right)+b\left(q^{(1)}-q^{(2)}\right)>0 .
$$

Now applying (8) and invoking Theorem 4, we obtain

Theorem 5. Cooperation is favored $\left(\rho_{\mathrm{C}}>\rho_{\mathrm{D}}\right)$ in the Prisoners'Dilemma game (13) on a weighted vertex-transitive graph $G$ of size $N$, with $B D, P C$, or DB-D updating and no mutation, for all sufficiently small $\delta>0$, if and only if

$$
-c(N-1)+b\left(N p^{(1)}-1\right)>0 .
$$

Recalling that $p^{(1)}$ is the edge weight of self-loops in $G$, we note that cooperation is never favored if $p^{(1)}=0$ :

Corollary 6. For the Prisoners' Dilemma game (13) on a weighted vertex-transitive graph $G$ with no self-loops $\left(p^{(1)}=0\right)$, with $B D, P C$, or DB-D updating and no mutation, cooperation is disfavored $\left(\rho_{\mathrm{C}}<\rho_{\mathrm{D}}\right)$ for all sufficiently small $\delta>0$.

A necessary condition for cooperation to be favored, which is approximately sufficient for large populations $(N \gg 1)$, is $b p^{(1)}>c$. This means that the benefit $b p^{(1)}$ that a cooperator gives to itself via self-interaction must exceed the $\cos t c$. Thus for BD, PC, and DB-D updating, cooperation is favored only if self-loops make this cooperation self-serving rather than costly.

4.2.2. Death-Birth and BD-D updating. For DB or BD-D updating, Condition (11) reduces to $f^{(0)}>f^{(2)}$. That is, cooperation is favored if and only if the focal cooperator has greater payoff than its neighbors-of-neighbors, on average. By Eq. (14), this condition is equivalent to

$$
-c\left(q^{(0)}-q^{(2)}\right)+b\left(q^{(1)}-q^{(3)}\right)>0 .
$$

Applying Eq. (9) twice to the respective coefficients of $b$ and $c$, we obtain

Theorem 7. Cooperation is favored $\left(\rho_{\mathrm{C}}>\rho_{\mathrm{D}}\right)$ in the Prisoners' Dilemma game (13) on a weighted vertex-transitive graph with $D B$ or BD-D updating and no mutation, for all sufficiently small $\delta>0$, if and only if

$$
-c\left(N+N p^{(1)}-2\right)+b\left(N p^{(1)}+N p^{(2)}-2\right)>0 .
$$

We note that $p^{(2)}=\sum_{k \in G} e_{i k}^{2}=\kappa^{-1}$, where $\kappa$ is the Simpson degree of $G$. Thus for graphs with no self-loops, we obtain 
Corollary 8. Cooperation is favored $\left(\rho_{\mathrm{C}}>\rho_{\mathrm{D}}\right)$ for the Prisoners' Dilemma game (13) on a weighted vertex-transitive graph with no self-loops, with $D B$ or $B D-D$ updating and no mutation, for all sufficiently small $\delta>0$, if and only if

$$
\frac{b}{c}>\frac{N-2}{N / \kappa-2} .
$$

For large population size, this condition becomes approximately $b / c>\kappa$, a generalization of the $b / c>k$ rule [68] to weighted graphs. In the case of unweighted graphs, substituting $\kappa=k$ yields the condition

$$
\frac{b}{c}>\frac{N-2}{N / k-2},
$$

a finite-population correction to the $b / c>k$ rule obtained by Taylor et al. [110] and Chen [14].

4.2.3. Imitation updating. For IM updating on an unweighted vertex-transitive graph of degree $k$, Condition (11) reduces to

$$
f^{(0)}>\frac{2}{k+2} f^{(1)}+\frac{k}{k+2} f^{(2)} .
$$

Applying Eqs. (14) and (9), we obtain

Theorem 9. Cooperation is favored $\left(\rho_{\mathrm{C}}>\rho_{\mathrm{D}}\right)$ for the Prisoners' Dilemma game (13) on a vertex-transitive graph with IM updating and no mutation, for all sufficiently small $\delta>0$, if and only if

$$
\frac{b}{c}>\frac{N(k+2)-2 k-2}{N-2 k-2} .
$$

In fact, this result holds for all regular graphs, not only vertex-transitive graphs [14]. For large populations, this condition becomes approximately $b / c>k+2$, a result obtained by Ohtsuki et al. [68] using pair approximation.

4.3. Arbitrary $2 \times 2$ matrix games. We now move to general $2 \times 2$ matrix games of the form (1), retaining the strategy labels $\mathrm{C}$ and $\mathrm{D}$ for convenience. A powerful theorem of Tarnita et al. [106] allows conditions for success in arbitrary $2 \times 2$ games to be determined from analysis of the simplified game (13). This theorem guarantees that, for a broad class of evolutionary processes based on games of the form (1), there is a "structure coefficient" $\sigma$, independent of $R, S, T$, and $P$, such that strategy $\mathrm{C}$ is favored if and only if $\sigma R+S>T+\sigma P$. Here we state this theorem for Markov chains on $\{\mathrm{C}, \mathrm{D}\}^{N}$, although the original result applies to a more general class of processes. 
Theorem 10 ([106]). For fixed $N$ and for each $\boldsymbol{s}, \boldsymbol{s}^{\prime} \in\{\mathrm{C}, \mathrm{D}\}^{N}$, let $p_{\boldsymbol{s} \rightarrow \boldsymbol{s}^{\prime}}(\mathbf{M})$ be differentiable, real-valued functions of a $2 \times 2$ matrix $\mathbf{M}$ with the following properties:

(i) The collection $\left\{p_{\boldsymbol{s} \rightarrow \boldsymbol{s}^{\prime}}(\mathbf{M})\right\}_{\boldsymbol{s}, \boldsymbol{s}^{\prime} \in\{\mathrm{C}, \mathrm{D}\}^{N}}$ are transition probabilities of an ergodic Markov chain on $\{\mathrm{C}, \mathrm{D}\}^{N}$ for each $\mathbf{M}$.

(ii) For $s \in\{\mathrm{C}, \mathrm{D}\}^{N}$, let $\overline{\boldsymbol{s}} \in\{\mathrm{C}, \mathrm{D}\}^{N}$ be the result of replacing each $\mathrm{C}$ with $\mathrm{D}$, and vice versa, in $\mathrm{s}$. Then

$$
p_{\boldsymbol{s} \rightarrow \boldsymbol{s}^{\prime}}\left(\begin{array}{ll}
a_{11} & a_{12} \\
a_{21} & a_{22}
\end{array}\right)=p_{\overline{\mathbf{s}} \rightarrow \overline{\boldsymbol{s}}^{\prime}}\left(\begin{array}{ll}
a_{22} & a_{21} \\
a_{12} & a_{11}
\end{array}\right) .
$$

(iii) For $\mathbf{M}=\left(\begin{array}{ll}0 & 1 \\ 0 & 0\end{array}\right),\langle x\rangle>\frac{1}{2}$, where $x$ is defined as in Eq. (10) and the brackets indicate expectation under the stationary distribution.

Then there exists a real number $\sigma$ such that, for any $R, S, T, P \in \mathbb{R}$, the Markov chain defined by $\left\{p_{\boldsymbol{s} \rightarrow \boldsymbol{s}^{\prime}}\left(\begin{array}{cc}\delta R & \delta S \\ \delta T & \delta P\end{array}\right)\right\}_{\boldsymbol{s}, \boldsymbol{s}^{\prime}}$, has the property that $\langle x\rangle>1 / 2$ for all sufficiently small $\delta>0$ if and only if $\sigma R+S>T+\sigma P$.

Condition (ii) above asserts that the process is symmetric with respect to interchanging the strategy labels $\mathrm{C}$ and $\mathrm{D}$. Condition (iii) ensures that $\mathrm{C}$ has greater expected frequency than $\mathrm{D}$ in a game that trivially favors $\mathrm{C}$.

In the case of evolutionary dynamics on graphs, $\sigma$ depends on the graph, update rule, and mutation rate, but not on the game. The advantage of this result is that the value of $\sigma-$ and therefore the success condition for an arbitrary game of the form (1) - can be obtained by considering only a restricted family of games such as the simplified Prisoner's Dilemma (13). In particular, combining Theorems 4, 5, 7, 9 and 10, we have

Corollary 11. Strategy $\mathrm{C}$ is favored $\left(\rho_{\mathrm{C}}>\rho_{\mathrm{D}}\right)$ in the game (1) on a weighted vertextransitive graph with no mutation, for all sufficiently small $\delta>0$, if and only if $\sigma R+S>T+\sigma P$, where

$$
\sigma= \begin{cases}\frac{1+p^{(1)}-\frac{2}{N}}{1-p^{(1)}} & \text { for BD, PC, or DB-D updating, } \\ \frac{1+2 p^{(1)}+p^{(2)}-\frac{4}{N}}{1-p^{(2)}} & \text { for DB or BD-D updating. }\end{cases}
$$

In particular, if the graph has no self loops,

$$
\sigma= \begin{cases}\frac{N-2}{N} & \text { for } B D, P C, \text { or DB-D updating, } \\ \frac{\kappa+1-\frac{4 \kappa}{N}}{\kappa-1} & \text { for } D B \text { or } B D-D \text { updating. }\end{cases}
$$

For IM updating on an unweighted vertex-transitive graph, the same result holds with

$$
\sigma=\frac{k+3-\frac{4(k+1)}{N}}{k+1} .
$$




\section{Nonzero mutation}

We now study games on graphs with mutation. This mutation may be genetic, or it may represent random strategy exploration in a social learning model [112]. High rates of mutation dilute the assortment of like types. This dilution can thwart cooperative strategies whose success depends on spatial clustering [5], [114].

5.1. Generating function expressions for IBD probabilities. We first derive expressions for IBD probabilities that are valid for arbitrary mutation rates. These expressions are in terms of the random walk generating function (or Green's function)

$$
\mathcal{E}_{i j}(z)=\sum_{n=0}^{\infty} p_{i j}^{(n)} z^{n}
$$

Montroll and Weiss [56] provide a general method for obtaining $\mathscr{E}_{i j}$ on lattices (see also [46]). We use $\mathcal{E}$ as a shorthand for $\mathcal{E}_{i i}$ (which, by vertex-transitivity, does not depend on $i$ ).

Lemma 12. For all $0<u<1$ and $i, j \in G$,

$$
q_{i j}=\frac{\mathcal{E}_{i j}(1-u)}{\mathcal{E}(1-u)} .
$$

Lemma 13. For all $0<u<1$ and $n \geq 0$,

$$
q^{(n+1)}=\frac{q^{(n)}-p^{(n)}(\mathcal{E}(1-u))^{-1}}{1-u}
$$

In particular,

$$
q^{(1)}=\frac{1-(\mathcal{E}(1-u))^{-1}}{1-u} .
$$

The expected IBD probability for two vertices chosen randomly with replacement is

$$
\bar{q}=\frac{1}{N u \mathscr{E}(1-u)} .
$$

For nonzero mutation rates, we use $\langle x\rangle>\frac{1}{2}$ as our criterion for success. By Theorem 4 this criterion reduces to Condition (12) under weak selection. We now obtain conditions for strategy selection under BD and DB updating; other update rules may be analyzed similarly. 
5.2. Birth-Death. For BD updating, Condition (12) for evolutionary success with mutation becomes

$$
(1-u)\left\langle\frac{\partial}{\partial \delta} \frac{F_{0}}{\sum_{j \in G} F_{j}}\right\rangle_{\substack{\delta=0 \\ s_{0}=\mathrm{C}}}-\left\langle\frac{\partial}{\partial \delta} \sum_{i \in G} \frac{e_{i 0} F_{i}}{\sum_{j \in G} F_{j}}\right\rangle_{\substack{\delta=0 \\ s_{0}=\mathrm{C}}}>0 .
$$

This condition reduces to $f^{(0)}-f^{(1)}-u\left(f^{(0)}-\bar{f}\right)>0$, where $\bar{f}$ is the average payoff over all vertices. (The term with $u$ represents mutational bias due to differential birth rates; see Section 4.1.) Upon substituting $\bar{f}=(-c+b)(1+\bar{q}) / 2$ and applying Eq. (14), we obtain

Theorem 14. On a weighted vertex-transitive graph with BD updating and mutation rate $0<u<1$, cooperation is favored $\left(\langle x\rangle>\frac{1}{2}\right)$ in the Prisoners' Dilemma game (13) for all sufficiently small $\delta>0$, if and only if

$$
-c\left(1-q^{(1)}-u(1-\bar{q})\right)+b\left(q^{(1)}-q^{(2)}-u\left(q^{(1)}-\bar{q}\right)\right)>0 .
$$

Strategy $\mathrm{C}$ is favored in the general game (1) for all sufficiently small $\delta>0$ if and only if $\sigma R+S>T+\sigma P$, where

$$
\sigma=\frac{1-q^{(2)}-u\left(1+q^{(1)}-2 \bar{q}\right)}{1-2 q^{(1)}+q^{(2)}-u\left(1-q^{(1)}\right)} .
$$

The values of $q^{(n)}$ and $\bar{q}$ - and hence the conditions for success - can be obtained for any particular graph $G$ using Lemma 13.

5.3. Death-Birth. For DB updating, Condition (12) reduces to $f^{(0)}>f^{(2)}$. Applying Eqs. (14) and (9) yields:

Theorem 15. On a weighted vertex-transitive graph with DB updating and mutation rate $0<u<1$, cooperation is favored $\left(\langle x\rangle>\frac{1}{2}\right)$ in the Prisoners' Dilemma game (13) for all sufficiently small $\delta>0$, if and only if

$$
-c\left(1-q^{(2)}\right)+b\left(q^{(1)}-q^{(3)}\right)>0 .
$$

Strategy $\mathrm{C}$ is favored for the general game (1) for all sufficiently small $\delta>0$ if and only if $\sigma R+S>T+\sigma P$, where

$$
\sigma=\frac{1+q^{(1)}-q^{(2)}-q^{(3)}}{1-q^{(1)}-q^{(2)}+q^{(3)}}
$$

Allen et al. [5] examined the dependence of Condition (19) on $u$ for a variety of graph families. In each case, the critical $b / c$ ratio for the success of cooperation increases with $u$, confirming the intuition that mutation impedes cooperation by diluting the spatial assortment of cooperators. 


\section{Different interaction and replacement graphs}

It is sometimes natural to consider different sets of edge weights for replacement and interaction [72], [110], [73]. This means that the frequency of game interaction between vertices $i$ and $j$ may differ from the frequency with which their occupants can replace each other.

The population structure in this case is described by a pair of graphs with the same vertex set $V$ : the replacement graph $G_{\mathrm{R}}$, with edge weights $\left\{e_{i j}\right\}_{i, j}$, and the interaction graph $G_{\mathrm{I}}$ with edge weights $\left\{w_{i j}\right\}_{i, j}$. Each time-step, each individual $i \in$ $V$ plays the game with its neighbors in $G_{\mathrm{I}}$, and retains the $w$-weighted average payoff $f_{i}$ from these interactions, rescaled to $F_{i}=F\left(\delta f_{i}\right)$. Once payoffs are obtained, the update rule (BD or $\mathrm{DB})$ is applied as described above, using the replacement graph $G_{\mathrm{R}}$.

We require that the graphs $G_{\mathrm{R}}$ and $G_{\mathrm{I}}$ have joint transitive symmetry. This means that for every pair of vertices $i, j \in V$, there is a bijection $T$ of $V$ such that $T(i)=j$ and, for every pair $h, \ell \in V, w_{T(h) T(\ell)}=w_{h \ell}$ and $e_{T(h) T(\ell)}=e_{h \ell}$. (In other words, for every pair $i, j \in V$, there is an isomorphism $T$ of both $G_{\mathrm{R}}$ and $G_{\mathrm{I}}$ that maps $i$ to $j$.) We also require that the adjacency matrices of $G_{\mathrm{R}}$ and $G_{\mathrm{I}}$ commute.

We define a $(n, m)$-random walk to be a random walk with $n$ steps taken using the weights $\left\{e_{i j}\right\}$ of $G_{\mathrm{R}}$, and $m$ steps taken using the weights $\left\{w_{i j}\right\}$ of $G_{\mathrm{I}}$. By commutativity of the adjacency matrices, the order in which these steps are taken does not affect the probability of termination at a particular vertex. We define $p^{(n, m)}$ as the probability that such a random walk terminates at its starting vertex, and $q^{(n, m)}$ as the stationary probability that it terminates at a vertex IBD to the initial vertex. Translating our earlier results into this notation, we have, for $0<u<1$,

$$
\begin{aligned}
q^{(n, 0)} & =p^{(n, 0)}+\left(q^{(n+1,0)}-p^{(n, 0)} q^{(1,0)}\right)(1-u), \\
q^{(1,0)} & =\frac{1-N u \bar{q}}{1-u}=\frac{1-[\mathscr{E}(1-u)]^{-1}}{1-u}, \\
q^{(n, 0)}-q^{(n+1,0)} & =u\left(N p^{(n, 0)}-1\right)+O\left(u^{2}\right) .
\end{aligned}
$$

Furthermore, a straightforward variation on the proof of Lemma 2 shows that

$$
q^{(n, m)}=p^{(n, m)}+\left(q^{(n+1, m)}-p^{(n, m)} q^{(1,0)}\right)(1-u),
$$

which admits the low-mutation expansion

$$
q^{(n, m)}-q^{(n+1, m)}=u\left(N p^{(n, m)}-1\right)+\mathcal{O}\left(u^{2}\right) .
$$

We again choose a focal node 0 , and consider the stationary distribution conditioned on 0 being a cooperator. In the case of the simplified Prisoner's Dilemma game (13), the expected payoff $f^{(n, 0)}$ at the terminus of an $(n, 0)$-random walk from 0 is

$$
f^{(n, 0)}=\frac{1}{2}\left(-c+b-c q^{(n, 0)}+b q^{(n, 1)}\right) .
$$


6.1. Birth-Death, pairwise comparison, and DB-D updating. Following the arguments of Section 4.2.1, we obtain that cooperation is favored under BD, PC, or DB-D updating if and only if $f^{(0,0)}>f^{(1,0)}$. This means that the focal cooperator must have greater payoff, on average, than its neighbors in the replacement graph. This condition yields the following result:

Theorem 16. For any pair $\left(G_{\mathrm{R}}, G_{\mathrm{I}}\right)$ of replacement and interaction graphs, with BD, $P C$, or DB-D updating and no mutation, cooperation is favored $\left(\rho_{\mathrm{C}}>\rho_{\mathrm{D}}\right)$ for the Prisoners' Dilemma game (13) for all sufficiently small $\delta>0$, if and only if

$$
-c(N-1)+b\left(N p^{(0,1)}-1\right)>0 .
$$

If $G_{\mathrm{I}}$ has no self-loops $\left(p^{(0,1)}=0\right)$, cooperation is disfavored $\left(\rho_{\mathrm{C}}<\rho_{\mathrm{D}}\right)$ for the Prisoners' Dilemma game (13) for all sufficiently small $\delta>0$. Strategy $\mathrm{C}$ is favored in the general game (1) for all sufficiently small $\delta>0$ if and only if $\sigma R+S>T+\sigma P$, where

$$
\sigma=\frac{1+p^{(0,1)}-\frac{2}{N}}{1-p^{(0,1)}}
$$

For large populations, the condition for success in the Prisoners' Dilemma game (13) is approximately $b p^{(0,1)}>c$. Thus we again find that, under BD updating, cooperation is only favored if the direct benefit to the cooperator, $b p^{(0,1)}$, outweighs the cost $c$.

In the case of positive mutation rates and $\mathrm{BD}$ updating, Condition (12) becomes $(1-u) f^{(0,0)}>f^{(1,0)}-u \bar{f}$, yielding:

Theorem 17. For any pair $\left(G_{\mathrm{R}}, G_{\mathrm{I}}\right)$ of replacement and interaction graphs, with $B D$ updating and mutation rate $0<u<1$, cooperation is favored $\left(\langle x\rangle>\frac{1}{2}\right)$ in the Prisoners' Dilemma game (13) for all sufficiently small $\delta>0$, if and only if

$$
-c\left(1-q^{(1,0)}-u(1-\bar{q})\right)+b\left(q^{(0,1)}-q^{(1,1)}-u\left(q^{(0,1)}-\bar{q}\right)\right)>0 .
$$

Strategy $\mathrm{C}$ is favored in the general game (1) for all sufficiently small $\delta>0$ if and only if $\sigma R+S>T+\sigma P$, where

$$
\sigma=\frac{1-q^{(1,0)}+q^{(0,1)}-q^{(1,1)}-u\left(1+q^{(0,1)}-2 \bar{q}\right)}{1-q^{(1,0)}-q^{(0,1)}+q^{(1,1)}-u\left(1-q^{(0,1)}\right)}
$$

6.2. Death-Birth and BD-D updating. For DB or BD-D updating with no mutation, Condition (11) becomes $f^{(0,0)}>f^{(2,0)}$, yielding:

Theorem 18. For any pair $\left(G_{\mathrm{R}}, G_{\mathrm{I}}\right)$ of replacement and interaction graphs, with $D B$ or BD-D updating and no mutation, cooperation is favored $\left(\rho_{\mathrm{C}}>\rho_{\mathrm{D}}\right)$ in the Prisoners' Dilemma game (13) for all sufficiently small $\delta>0$, if and only if

$$
-c\left(N+N p^{(1,0)}-2\right)+b\left(N p^{(0,1)}+N p^{(1,1)}-2\right)>0 .
$$


Strategy $\mathrm{C}$ is favored in the general game (1) for all sufficiently small $\delta>0$ if and only if $\sigma R+S>T+\sigma P$, where

$$
\sigma=\frac{1+p^{(1,0)}+p^{(0,1)}+p^{(1,1)}-\frac{4}{N}}{1+p^{(1,0)}-p^{(0,1)}-p^{(1,1)}} .
$$

For large populations with no self-loops in $G_{\mathrm{R}}$ or $G_{\mathrm{I}}$, Condition (20) becomes approximately $b p^{(1,1)}>c$. This result was obtained by Ohtsuki et al. [73], [72] (using pair approximation) in the case that the interaction and replacement graphs are unweighted.

For positive mutation rates and DB updating, Condition (12) reduces to $f^{(0,0)}>$ $f^{(2,0)}$, yielding:

Theorem 19. For any pair $\left(G_{\mathrm{R}}, G_{\mathrm{I}}\right)$ of replacement and interaction graphs, with $D B$ updating and mutation rate $0<u<1$, cooperation is favored $\left(\langle x\rangle>\frac{1}{2}\right)$ in the Prisoners' Dilemma game (13) for all suffciently small $\delta>0$, if and only if

$$
-c\left(1-q^{(2,0)}\right)+b\left(q^{(0,1)}-q^{(2,1)}\right)>0 .
$$

Strategy $\mathrm{C}$ is favored in the general game (1) for all suffciently small $\delta>0$ if and only if $\sigma R+S>T+\sigma P$, where

$$
\sigma=\frac{1+q^{(0,1)}-q^{(2,0)}-q^{(2,1)}}{1-q^{(0,1)}-q^{(2,0)}+q^{(2,1)}} .
$$

6.3. Imitation. For IM updating with no mutation, Condition (11) reduces to

$$
f^{(0)}>\frac{2}{k_{\mathrm{R}}+2} f^{(1)}+\frac{k_{\mathrm{R}}}{k_{\mathrm{R}}+2} f^{(2)},
$$

where $k_{\mathrm{R}}$ is the degree of the replacement graph. This leads to:

Theorem 20. For any pair $\left(G_{\mathrm{R}}, G_{\mathrm{I}}\right)$ of unweighted replacement and interaction graphs, with IM updating and no mutation, cooperation is favored $\left(\rho_{\mathrm{C}}>\rho_{\mathrm{D}}\right)$ in the Prisoners' Dilemma game (13) for all sufficiently small $\delta>0$, if and only if

$$
-c\left(N\left(k_{\mathrm{R}}+2\right)-2 k_{\mathrm{R}}-2\right)+b\left(N k_{\mathrm{R}} p^{(1,1)}-2 k_{\mathrm{R}}-2\right)>0 .
$$

Strategy $\mathrm{C}$ is favored in the general game (1) for all sufficiently small $\delta>0$ if and only if $\sigma R+S>T+\sigma P$, where

$$
\sigma=\frac{k_{\mathrm{R}}+2+k_{\mathrm{R}} p^{(1,1)}-\frac{4\left(k_{\mathrm{R}}+1\right)}{N}}{k_{\mathrm{R}}+2-k_{\mathrm{R}} p^{(1,1)}} .
$$

\section{Extensions}

We now briefly review the many extensions of this theory that have been considered, highlighting general themes and directions for future investigation. 
7.1. Other instances of the $\boldsymbol{b} / \boldsymbol{c}>\boldsymbol{k}$ rule. The condition $b / c>k$ elegantly expresses how spatial structure can promote the evolution of cooperation. If the benefitto-cost ratio exceeds the number of neighbors per individual, then the advantage cooperators gain through assortment outweighs the harm of exploitation by defectors. The precise statement (a special case of Corollary 8) is that, on unweighted vertextransitive graphs of degree $k$ with $\mathrm{DB}$ or BD-D updating, cooperation is favored in the Prisoners' Dilemma game (13) for sufficiently weak selection if and only if

$$
\frac{b}{c}>\frac{N-2}{N / k-2} \underset{N \rightarrow \infty}{\longrightarrow} \frac{b}{c}>k .
$$

This rule also applies in a number of cases not covered by the mathematical framework developed above. Chen [14] showed that Condition (21) holds for all finite $k$-regular graphs, not only those that are vertex-transitive. Cox, Durrett and Perkins [16] proved that the $b / c>k$ rule also holds for infinite lattices $\mathbb{Z}^{d}$ of dimension $d \geq 3$. (In this case, infinite population size requires that fixation probability be replaced by a suitable notion of "taking over".)

7.2. Update rules and the fate of cooperation. One of the most intriguing aspects of spatial evolutionary game theory is the effect of the update rule. As we have seen, the conditions for success in the absence of mutation reduce to $f^{(0)}>f^{(1)}$ for BD, PC, and DB-D updating; $f^{(0)}>f^{(2)}$ for DB and BD-D updating; and $f^{(0)}>\frac{2}{k+2} f^{(1)}+\frac{k}{k+1} f^{(2)}$ for IM updating. In each case, the payoff of the focal cooperator is compared to a weighted average of payoffs at various distances. The weighting depends on the update rule.

For update rules leading to the $f^{(0)}>f^{(1)}$ condition, cooperation cannot be favored in the Prisoners' Dilemma game (13), except in cases where self-loops allow for cooperation to be self-serving. This result is often explained (e.g. [49]) in terms of scales of interaction and competition. The condition $f^{(0)}>f^{(1)}$ suggests an indirect competition for space at distance one, the same scale as game interaction. The concurrence of these scales is said to cancel the positive effects of cooperation. However, given that $\mathrm{BD}$ and similar update rules provide no benefit to (non-self-serving) cooperation even when the replacement and interaction graphs differ (Theorem 16), this explanation appears incomplete. From the derivation of Theorem 5, this effect appears more directly due to the fact that $q^{(1)}<q^{(2)}$ (or $q^{(0,1)}<q^{(1,1)}$ in the case $G_{\mathrm{R}} \neq G_{\mathrm{I}}$ ) for graphs with no self-loops, which implies that the immediate neighbors of the focal cooperator receive more benefit than the focal cooperator does. For update rules leading to $f^{(0)}>f^{(2)}$, the success of cooperation (for sufficiently large $b / c$ ) results from the fact that $q^{(1)}>q^{(3)}$ (or $q^{(0,1)}>q^{(2,1)}$ ) in the absence of self-loops, from which it follows that the focal cooperator receives more benefit than do its neighbors at distance two. 
7.3. Evolutionary stability and replicator dynamics. Evolutionary game theory was formulated in the context of well-mixed (non-spatial) populations. It is therefore interesting to look for spatial analogues of fundamental concepts arising in non-spatial evolutionary game theory.

One such concept is evolutionary stability. An evolutionarily stable strategy (ESS) [54], [53], [37] is a strategy over which no sufficiently rare alternative strategy has an advantage. For the $2 \times 2$ matrix game

$$
\begin{gathered}
\mathrm{A} \\
\mathrm{A} \\
\mathrm{B}
\end{gathered}\left(\begin{array}{ll}
a & b \\
c & d
\end{array}\right),
$$

strategy A is evolutionarily stable if and only if

$$
(a>c) \text { or }(a=c \text { and } b>d) .
$$

In particular, $a>c$ is sufficient for the evolutionary stability of A. Ohtsuki and Nowak [71] used pair approximation to obtain analogues of the $a>c$ condition on graphs. For example, they found that, with DB updating, the condition

$$
\left(k^{2}-1\right) a+b>\left(k^{2}-k-1\right) c+(k+1) d
$$

is sufficient for the evolutionary stability of A under weak selection (and is necessary except in the nongeneric case that equality holds).

Another fundamental concept of non-spatial evolutionary game theory is replicator dynamics, a system of ordinary differential equations describing strategy frequencies over time, assuming reproductive rates proportional to game payoff [111], [37], [38], [66]. Ohtsuki and Nowak [70], again using pair approximation, derived spatial analogues of these equations on graphs under various update rules. A more rigorous spatial analogue of replicator dynamics was provided by Cox, Durrett, and Perkins [16] in their study of evolutionary dynamics on square lattices of dimension $\geq 3$. They showed that, under a sequence of renormalizations of space and time, the dynamics of local strategy densities converge to a solution to a certain partial differential equation. This PDE describes how strategies propagate through space, and can be interpreted as a spatial replicator equation.

7.4. Different games affecting birth and death. The interactions that affect reproduction in a given population may differ from those affecting survival. To explore the evolutionary consequences of such a difference, Débarre et al. [20] developed a general framework in which birth and death probabilities may depend on different games. They obtained a generalization of Theorems 16 and 18 in which there are two values of $\sigma$ : one for the game affecting birth and another for the game affecting death. 
7.5. Non-weak selection. The results proven here hold for weak selection - that is, they are valid only for sufficiently small $\delta>0$. This is a natural regime to consider, as it describes the case that game interactions comprise only a small component of an individual's overall fitness. However, there are many scenarios in biological and cultural evolution in which interactions have a large effect on fitness. It is therefore desirable to obtain results that are valid beyond the weak selection regime.

Non-weak selection presents new mathematical difficulties, since the dynamics can no longer be viewed as a perturbation of neutral drift. Exact results have only been obtained for very simple graph structures such as the cycle [69] and the star [11], [35] (Figure 4). Non-weak selection is represented by using the game payoffs $f_{i}$ directly - rather than their rescalings $F_{i}=F\left(\delta f_{i}\right)$ - to determine birth and death

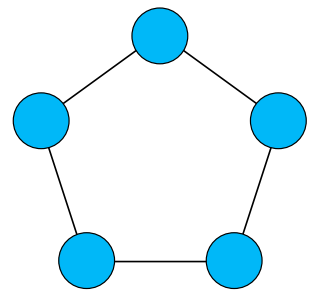

Cycle

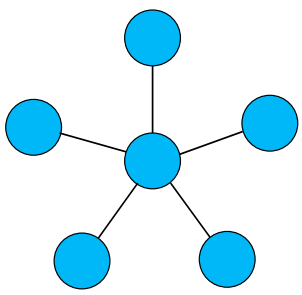

Star

Figure 4. The cycle (one-dimensional periodic lattice) and the star are two simple graph structures for which exact conditions for evolutionary success under non-weak selection can be obtained.

probabilities according to the update rule. (Note that this approach requires that the entries of the payoff matrix be nonnegative; thus it applies to the general game (1) with $R, S, T, P \geq 0$ but not to the simplified Prisoners' Dilemma game (13)). For the cycle with DB updating and in the limit $N \rightarrow \infty$, cooperation is favored if and only if $R(R+S)>P(P+T)$ [69]. For BD updating, the condition is $R+S>T+P$, which is the same as the condition for success in large well-mixed populations [108], [65], [39], and which is never satisfied for Prisoners' Dilemma games $(T>R>P>S)$.

For other graph structures, our understanding of evolutionary game dynamics with non-weak selection comes primarily from simulations [36], [95], [82], [78]. For example, Roca et al. [81], [82] performed Monte Carlo simulations of cooperation games on lattices and random regular graphs, systematically varying the game parameters, graph degree, and update rule. Their major finding is that the effects of spatial structure on the evolution of cooperation depend strongly on both the game and the update rule, in a way that is not easily summarized in any general law. 
7.6. Asymmetric graphs. Biological and social networks are often strongly asymmetric [93], [1], [58]. It is therefore of great interest to understand strategy selection for games on asymmetric graphs. Exact analysis is difficult beyond simple cases such as the star graph [11], [35], but extensive simulation results are available [84], [28], [95, 7], [78], [120].

A well-known simulation study of Santos et al. [84] shows that scale-free networks [93], [1], [58] with PC updating strongly promote cooperation in Prisoners' Dilemma and Snowdrift games. However, Tomohiko [44] found (using pair approximation and simulation) that for DB updating and weak selection on asymmetric graphs, the $b / c>k$ condition generalizes to $b / c>\left\langle k_{\mathrm{nn}}\right\rangle$, where $\left\langle k_{\mathrm{nn}}\right\rangle$ is the expected average degree among nearest neighbors of a randomly chosen vertex. For fixed average degree $\langle k\rangle,\left\langle k_{\mathrm{nn}}\right\rangle$ increases with degree heterogeneity. In the idealized case of infinite, degree-uncorrelated scale-free graphs, $\left\langle k_{\mathrm{nn}}\right\rangle$ is infinite and cooperation is never favored in the Prisoner's Dilemma game (13). Thus the question of whether scale-free networks promote cooperation is strongly dependent on the update rule.

7.7. Multi-strategy games. So far we have considered only games with two strategies. Many other types of interactions can be modeled using games with more than two strategies, represented by $n \times n$ matrices.

One particularly interesting class of multi-strategy games is rock-paper-scissors games, in which each of three strategies bests another and is bested by the third. On two-dimensional lattices, simulations show the system typically settles into a dynamic pattern in which the three strategies persist and continually invade each others' territory [102], [103], [104]. For other graph structures, a different dynamical behavior is possible, in which the population spends increasing amounts of time dominated by each strategy in sequence [102], [103], [86], [97], [101], similar to the convergence to heteroclinic attractors found in the case of well-mixed populations [52], [37], [38]. These results are reviewed in depth by Szabo and Fath [95].

Another important multi-strategy game is the Prisoners' Dilemma with reactive strategies. In this case, the Prisoners' Dilemma game is played repeatedly (often with a constant continuation probability per round), and each player's choice may depend on what has happened in previous rounds. The best-known reactive strategy is Tit-forTat (TFT), in which a player initially plays $C$ and subsequently mirrors the opponent's choice in the previous round [8]. When paired against other TFT players or those who play only C (ALLC), both players receive the "reward" payoff $R$ each round; when paired against those who play only D (ALLD), both players receive the "punishment" payoff $P$ each round after the first (recall $T>R>P>S$ for Prisoners' Dilemma games). Simulation of the evolutionary dynamics of the strategies ALLC, ALLD, and TFT on lattices and random regular graphs reveals a variety of possible behaviors including fixation of ALLD, coexistence of only ALLC and ALLD, and coexistence of all three strategies - depending on the game parameters and graph topology [100]. 
The structure coefficient theorem [106], a crucial tool in our analysis of twostrategy games, can be generalized to games with $n$ strategies [107]. For a wide class of evolutionary processes based on $n \times n$ matrix games, the condition for the success of strategy $i$ takes the form

$$
\left(\sigma_{1} a_{i i}+\bar{a}_{i j}-\bar{a}_{j i}-\sigma_{1} \bar{a}_{j j}\right)+\sigma_{2}\left(\bar{a}_{i j}-\bar{a}_{j k}\right)>0 .
$$

Above, $a_{i j}$ are the elements of the payoff matrix, and the bars indicate arithmetic averages over all values of the indices $j$ and $k$. The structure coefficients $\sigma_{1}$ and $\sigma_{2}$ depend on the population structure, update rule, and mutation rate, but do not depend on the game matrix. Here success is defined as having expected frequency greater than $\frac{1}{n}$ in the mutation-selection stationary distribution. As in the $2 \times 2$ case, this result allows the condition for success in a general game to be obtained from analysis of special classes of games.

7.8. Multiplayer games. Many biological and social interactions involve more than two individuals. Such interactions can be modeled as multiplayer games. Payoffs in multiplayer games are described by multidimensional arrays in place of matrices. The evolutionary dynamics of strategies in such games exhibit a wide variety of behaviors even in well-mixed populations [26], [27]. Exact conditions for success in a general multiplayer game have been obtained in the case of a cycle [115], but analysis is difficult for more complex graph structures. The current state of the art in multiplayer games on graphs is reviewed by Perc et al. [76].

The most commonly studied multiplayer game is the public goods game, a social dilemma in which players choose whether or not to contribute to a public good. The overall payoff (an increasing function of the total contributions) is shared equally among all players. As in the Prisoners' Dilemma, noncooperation is the only evolutionarily stable strategy in a well-mixed population. In spatial versions [96], [85], the game is played among groups consisting of a vertex and its nearest neighbors. For PC updating, degree heterogeneity was found to have a positive effect on cooperation in public goods games [85]; however, as in other cases, this effect is likely dependent on the update rule.

One variation on public goods games is to suppose that the public good diffuses spatially, so that it is shared unequally depending on proximity to the producer [2], [10]. This mimics the sharing of public goods in microbial colonies [18], [41]. In this case, for DB updating, weak selection, and large population size, cooperation is favored if and only if $b / c>\left(\phi_{0}+\phi_{1}\right)^{-1}$, where $c$ is the cost of production, $b$ is the total benefit shared by all recipients, $\phi_{0}$ is the fraction of public goods retained by the producer, and $\phi_{1}$ is the average fraction of public goods retained by each of the producer's immediate neighbors [2].

7.9. Dynamic graphs. A final extension is to suppose that the graph itself may change along with the distribution of strategies. For example, social or spatial rela- 
tionships may change based on prior interactions [90], [23], [75], [74] reputations [25], or other factors [99], [98], [105], [119], [13]. While a great variety of models have been explored (as reviewed by Perc and Szolnoki [77]), a general theme is that cooperation is enhanced by mechanisms that allow cooperators to self-segregate, and hindered by mechanisms that break links between cooperators or allow defectors to infiltrate cooperator clusters.

7.10. Connections to empirical work. The inspiration for evolutionary games on graphs comes from genetic evolution in spatially structured populations, and from cultural evolution driven by social learning on networks. While the questions explored here are mathematically interesting in their own right, it is also important to ask what they reveal about the evolution of cooperation in nature and society.

The biological predictions of spatial evolutionary game theory can be tested via microorganism experiments. Microbes cooperate in a variety of ways, often by producing chemical public goods [18], [29]. A number of experiments [42], [15] have shown that group or patch structure, with limited dispersal between patches, can promote public goods cooperation. Fewer studies have investigated the effects of spatial structure directly. The evidence so far is that spatial structure can also promote cooperation [45], [19], [41], but this effect depends also on how widely the public good is shared [47]. It is also important to ask what kinds of update rules best describe evolution in microbial colonies [3].

Predictions regarding cultural evolution can be tested using human behavioral experiments, in which subjects interact via matrix games with their neighbors and can see their neighbors' strategies and payoffs. Experiments involving static graphs [114], [94], [31], [34] show little evidence so far of a spatial benefit to cooperation. There are a number of possible explanations: that the update rules used by subjects do not support cooperation [30], [34], that subjects have a high rate of strategy exploration (analogous to mutation) [114], or simply that the payoff matrices used in these experiments do not meet theoretically necessary thresholds for the success of cooperation (e.g. $b / c>k$ ) [80]. In contrast, experiments involving dynamic networks with active partner choice [24], [79], [117], [40] show a significant benefit to cooperation.

\section{Outlook}

Evolutionary game theory on graphs is a rich and elegant framework for studying how spatial population structure affects the evolution of social behavior. The conditions for evolutionary success are now well-understood for $2 \times 2$ games on graphs with symmetry under weak selection. However, there are many open problems involving asymmetric graphs, non-weak selection, and dynamic graphs. Identity-by-descent 
analysis, formalized via the IBD-enriched evolutionary Markov chain, is a powerful tool for studying spatial evolutionary dynamics in the weak selection regime.

As we have seen, the choice of update rule significantly affects the outcomes of evolutionary game competition. This has several implications for evolutionary theory. When modeling a specific evolutionary scenario, the update rule should be carefully chosen to correspond to the mechanisms of replacement in the population under study. For more abstract investigations, it is important to consider a variety of update rules in order to fully understand the evolutionary consequences of spatial structure. The strong dependence on update rules also highlights the need for mathematically general approaches to spatial evolution [106], [107], [4], [20], in which different update rules can be considered under a common framework.

Acknowledgements. We thank Krishnendu Chatterjee and Yu-Ting Chen for helpful comments on our manuscript. The Foundational Questions in Evolutionary Biology initiative at Harvard University is supported by a grant from the John Templeton Foundation.

\section{Appendix A. Proofs regarding identity-by-descent}

Lemma 1. For $0<u \leq 1$, $\widetilde{\mathcal{M}}$ has a unique stationary distribution $\left\{\pi_{(s, \iota)}\right\}_{(s, \iota)}$. For any states $(\boldsymbol{s}, \iota)$ and $\left(\boldsymbol{s}^{\prime}, \iota^{\prime}\right), \lim _{n \rightarrow \infty} p_{\left(\boldsymbol{s}^{\prime}, \iota^{\prime}\right) \rightarrow(\boldsymbol{s}, \iota)}^{(n)}=\pi_{(\boldsymbol{s}, l)}$. Any state $(\boldsymbol{s}, \iota)$ with $\pi_{(s, \iota)}>0$ has the property that $s_{i}=s_{j}$ for any pair $i, j \in G$ with $\iota_{i j}=1$.

Proof. Let $\left(s^{0}, \iota^{0}\right)$ be the state in which $s_{i}^{0}=\mathrm{C}$ for all $i \in G$ (all individuals have type C) and $\iota_{i j}^{0}=0$ for all $i \neq j$ (no two individuals are IBD). This state can be reached from any other state in at most $N$ steps (with each step involving a mutation to type $\mathrm{C}$ ). Thus $\widetilde{\mathcal{M}}$ has a single closed communicating class $C$ containing $\left(s^{0}, \iota^{0}\right)$, and all states not in $\mathcal{C}$ are transient. Thus $\tilde{\mathcal{M}}$ has a unique stationary distribution, supported in $\mathcal{C}$. Since the probability of transition from $\left(s^{0}, \iota^{0}\right)$ to itself is nonzero, the dynamics of $\widetilde{\mathcal{M}}$ restricted to $C$ are aperiodic, proving the convergence claim.

For the third claim, we let $\mathcal{I} \subset\{\mathrm{C}, \mathrm{D}\}^{N} \times \Pi_{G}$ denote the set of states of $\tilde{\mathcal{M}}$ that satisfy the desired property. Transitions out of the set $\mathcal{I}$ have zero probability according to the defined transition probabilities of $\widetilde{\mathcal{M}}$. Thus all recurrent states of $\widetilde{\mathcal{M}}$ are in $\mathcal{I}$, proving the claim.

Lemma 2. For all $0<u \leq 1$ and $n \geq 0$,

$$
q^{(n)}=p^{(n)}+\left(q^{(n+1)}-p^{(n)} q^{(1)}\right)(1-u) .
$$

Proof. In the main text we showed that the $q_{i j}$ satisfy the recurrence relations

$$
q_{i j}= \begin{cases}1 & \text { if } i=j, \\ \frac{1-u}{2} \sum_{k \in G}\left(e_{k i} q_{k j}+e_{k j} q_{k i}\right) & \text { if } i \neq j\end{cases}
$$


Combining these with the definition of $q^{(n)}$, Eq. (5), yields

$$
\begin{aligned}
q^{(n)} & =\frac{1}{N} \sum_{i, j \in G} p_{i j}^{(n)} q_{i j} \\
& =\frac{1}{N} \sum_{i \in G} p_{i i}^{(n)} q_{i i}+\frac{1-u}{2 N} \sum_{\substack{i, j \in G \\
i \neq j}} \sum_{k \in G} p_{i j}^{(n)}\left(e_{k i} q_{k j}+e_{k j} q_{k i}\right) \\
& =p^{(n)}+\frac{1-u}{2 N} \sum_{\substack{i, j, k \in G \\
i \neq j}} p_{i j}^{(n)} e_{k i} q_{k j}+\frac{1-u}{2 N} \sum_{\substack{i, j, k \in G \\
i \neq j}} p_{i j}^{(n)} e_{k j} q_{k i} .
\end{aligned}
$$

We now examine the second term:

$$
\begin{aligned}
\frac{1-u}{2 N} \sum_{\substack{i, j, k \in G \\
i \neq j}} p_{i j}^{(n)} e_{k i} q_{k j} & =\frac{1-u}{2 N} \sum_{j, k \in G}\left(\sum_{i \in G} e_{k i} p_{i j}^{(n)} q_{k j}-e_{k j} p_{j j}^{(n)} q_{k j}\right) \\
& =\frac{1-u}{2 N}\left(\sum_{j, k \in G} p_{k j}^{(n+1)} q_{k j}-p^{(n)} \sum_{j, k \in G} e_{k j} q_{k j}\right) \\
& =\frac{1-u}{2}\left(q^{(n+1)}-p^{(n)} q^{(1)}\right) .
\end{aligned}
$$

By a similar argument, the third term is also equal to $\frac{1-u}{2}\left(q^{(n+1)}-p^{(n)} q^{(1)}\right)$. This completes the proof.

Lemma 3. For all $0<u \leq 1$ and $i \in G$,
(i) $\operatorname{Pr}_{\delta=0}\left[s_{i}=\mathrm{C} \mid s_{0}=\mathrm{C}, \iota_{0 i}=1\right]=1$,
(ii) $\operatorname{Pr}_{\delta=0}\left[s_{i}=\mathrm{C} \mid s_{0}=\mathrm{C}, \iota_{0 i}=0\right]=\frac{1}{2}$.
(iii) $\operatorname{Pr}_{\delta=0}\left[s_{i}=\mathrm{C} \mid s_{0}=\mathrm{C}\right]=\frac{1+q_{0 i}}{2}$.

Proof. Claim (i) follows directly from Lemma 1. For Claim (ii), we consider the function $\phi:\{\mathrm{C}, \mathrm{D}\}^{N} \times \Pi_{G} \rightarrow\{\mathrm{C}, \mathrm{D}\}^{N} \times \Pi_{G}$ that switches the type of all individuals not IBD to 0 . That is, $\phi(s, \iota)=(\tilde{\boldsymbol{s}}, \iota)$ where, for all $i \in G$,

$$
\tilde{s}_{i}= \begin{cases}s_{i} & \text { if } \iota_{i 0}=1, \\ \mathrm{C} & \text { if } \iota_{i 0}=0 \text { and } s_{i}=\mathrm{D}, \\ \mathrm{D} & \text { if } \iota_{i 0}=0 \text { and } s_{i}=\mathrm{C} .\end{cases}
$$

Notice that the IBD relation $\iota$ is unchanged by the action of $\phi$. Since types C and D are interchangeable in the neutral drift $(\delta=0)$ case, $\phi$ commutes with the dynamics 
of $\tilde{\mathcal{M}}$ in the sense that $p_{\phi(s, \iota) \rightarrow \phi\left(s^{\prime}, \iota^{\prime}\right)}=p_{(s, \iota) \rightarrow\left(s^{\prime}, \iota^{\prime}\right)}$ for any pair of states $(s, \iota)$ and $\left(\boldsymbol{s}^{\prime}, \iota^{\prime}\right)$. Turning now to stationary probabilities, Lemma 1 implies that for any recurrent state $(s, \iota)$ of $\tilde{\mathcal{M}}$,

$$
\pi_{\phi(s, l)}=\lim _{n \rightarrow \infty} p_{\phi(s, \iota) \rightarrow \phi(s, l)}^{(n)}=\lim _{n \rightarrow \infty} p_{(s, \imath) \rightarrow(s, l)}^{(n)}=\pi_{(s, l)} .
$$

Thus stationary probabilities are also preserved by the action of $\phi$. It follows that for each fixed $i \in G$, the set $\left\{(s, \iota): s_{0}=\mathrm{C}, \iota_{0 i}=0, s_{i}=\mathrm{C}\right\}$ has the same stationary probability as the set $\left\{(s, \iota): s_{0}=\mathrm{C}, \iota_{0 i}=0, s_{i}=\mathrm{D}\right\}$, proving Claim (ii). Finally, Claim (iii) follows from combining Claims (i) and (ii) with the definition $q_{0 i}=\left\langle\iota_{0 i}\right\rangle_{\delta=0}$.

Lemma 12. For all $0<u<1$ and $i, j \in G$,

$$
q_{i j}=\frac{\mathcal{E}_{i j}(1-u)}{\mathcal{E}(1-u)} .
$$

Proof. The stationary IBD probabilities $q_{i j}$ are the unique solution to the recurrence relations (22). We show that the quotients $\left\{\mathscr{E}_{i j}(1-u) / \mathscr{E}(1-u)\right\}_{i, j}$ satisfy these recurrence relations and therefore are equal to $\left\{q_{i j}\right\}_{i, j}$. By definition we have that for $i=j$,

$$
\frac{\mathscr{E}_{i j}(1-u)}{\mathscr{E}(1-u)}=\frac{\mathscr{E}(1-u)}{\mathcal{E}(1-u)}=1 \text {. }
$$

In the case $i \neq j$,

$$
\begin{aligned}
& \frac{1-u}{2} \sum_{k \in G}\left(e_{k i} \frac{\mathscr{e}_{k j}(1-u)}{\mathscr{E}(1-u)}+e_{k j} \frac{\mathscr{E}_{k i}(1-u)}{\mathscr{E}(1-u)}\right) \\
= & \frac{1-u}{2 \mathscr{E}(1-u)}\left(\sum_{n=0}^{\infty} \sum_{k \in G} e_{i k} p_{k j}^{(n)}(1-u)^{n}+\sum_{n=0}^{\infty} \sum_{k \in G} e_{j k} p_{k i}^{(n)}(1-u)^{n}\right) \\
= & \frac{1}{2 \mathscr{E}(1-u)}\left(\sum_{n=0}^{\infty} p_{i j}^{(n+1)}(1-u)^{n+1}+\sum_{n=0}^{\infty} p_{j i}^{(n+1)}(1-u)^{n+1}\right) \\
= & \frac{\mathscr{E}_{i j}(1-u)}{\mathcal{E}(1-u)} .
\end{aligned}
$$

Above, in equating $\sum_{n=0}^{\infty} p_{i j}^{(n+1)}(1-u)^{n+1}$ with $\Theta_{i j}(1-u)$, we have made use of the fact that $p_{i j}^{(0)}=0$ for $i \neq j$, and thus the zeroth-order term of $\mathscr{S}_{i j}(1-u)$ is zero.

Lemma 13. For all $0<u<1$ and $n \geq 0$,

$$
q^{(n+1)}=\frac{q^{(n)}-p^{(n)}(\mathcal{E}(1-u))^{-1}}{1-u} .
$$


In particular,

$$
q^{(1)}=\frac{1-(\mathscr{E}(1-u))^{-1}}{1-u} .
$$

The expected IBD probability for two vertices chosen randomly with replacement is

$$
\bar{q}=\frac{1}{N u \mathscr{E}(1-u)} .
$$

Proof. We first obtain Eq. (25). For any vertex $i$, we write $q^{(1)}$ as

$$
\begin{aligned}
q^{(1)} & =\sum_{j \in G} e_{i j} q_{i j} \\
& =\frac{1}{\mathscr{E}(1-u)} \sum_{j \in G} e_{i j} \mathscr{G}_{i j}(1-u) \\
& =\frac{1}{\mathscr{E}(1-u)} \sum_{n=0}^{\infty} \sum_{j \in G} e_{i j} p_{i j}^{(n)}(1-u)^{n} \\
& =\frac{1}{(1-u) \mathscr{E}(1-u)} \sum_{n=0}^{\infty} p^{(n+1)}(1-u)^{n+1} \\
& =\frac{1}{(1-u) \mathscr{E}(1-u)}(\mathscr{E}(1-u)-1) \\
& =\frac{1-(\mathscr{E}(1-u))^{-1}}{1-u} .
\end{aligned}
$$

This verifies Eq. (25). For the case $n \geq 1$, we rewrite Eq. (6) as

$$
q^{(n+1)}=\frac{q^{(n)}-p^{(n)}}{1-u}+p^{(n)} q^{(1)} .
$$

Substituting for $q^{(1)}$ from Eq. (25) yields Eq. (24). Eq. (26) is obtained by substituting Eq. (25) into Eq. (7) and solving for $\bar{q}$, or alternatively, by averaging Eq. (23) over all $j \in G$ and using the series definition (17) for $\mathscr{G}_{i j}$.

\section{Appendix B. Proofs regarding evolutionary success}

Here we prove Theorem 4, which gives a general condition for evolutionary success under weak selection. We begin by considering the stationary distribution of $\mathcal{M}$ conditioned on both types being present. We recall that $\mathbf{C}$ and $\mathbf{D}$ denote the states of $\mathcal{M}$ consisting of all C's and all D's, respectively. For any $u, \delta$ with $0<u \leq 1$ and 
$\delta \geq 0$, the probability $\pi_{\boldsymbol{s} \mid \notin\{\mathbf{C}, \mathbf{D}\}}$ of a state $\boldsymbol{s} \in\{\mathrm{C}, \mathrm{D}\}^{N} \backslash\{\mathbf{C}, \mathbf{D}\}$ in this conditional stationary distribution is given by

$$
\pi_{\boldsymbol{s} \mid \notin\{\mathbf{C}, \mathbf{D}\}}(u, \delta)=\frac{\pi_{\boldsymbol{s}}(u, \delta)}{1-\pi_{\mathbf{C}}(u, \delta)-\pi_{\mathbf{D}}(u, \delta)} .
$$

Lemma 14. Let $\mathcal{M}_{u, \delta}$ denote the evolutionary Markov chain with fixed game, graph, and update rule, where the mutation rate $0<u \leq 1$ and selection strength $\delta>0$ are allowed to vary. Then $\pi_{\boldsymbol{s} \mid \notin\{\mathbf{C}, \mathbf{D}\}}(u, \delta)$ extends uniquely to a smooth, rational function of $(u, \delta) \in[0,1] \times[0, \infty)$.

Proof. We first note that, for all update rules considered here, the transition probabilities $p_{s \rightarrow s^{\prime}}(u, \delta)$ of $\mathcal{M}$ are smooth, rational functions of $(u, \delta) \in[0,1] \times[0, \infty)$.

Allen and Tarnita showed in the proof of Theorem 3 of [4] that, for all $(u, \delta) \in$ $(0,1] \times[0, \infty)$, the $\pi_{\boldsymbol{s} \mid \notin\{\mathbf{C}, \mathbf{D}\}}$ are stationary probabilities for a reduced Markov chain $\mathcal{M}_{\mid \notin\{\mathbf{C}, \mathbf{D}\}}$ on $\{\mathrm{C}, \mathrm{D}\}^{N} \backslash\{\mathbf{C}, \mathbf{D}\}$. The transition probability between states $\boldsymbol{s}$ and $\boldsymbol{s}^{\prime}$ of $\mathcal{M}_{\mid \notin\{\mathbf{C}, \mathbf{D}\}}$ is

$$
p_{\boldsymbol{s} \rightarrow \boldsymbol{s}^{\prime}}(u, \delta)+p_{\boldsymbol{s} \rightarrow \mathbf{C}}(u, \delta) r_{\mathbf{C} \rightarrow \boldsymbol{s}^{\prime}}(u, \delta)+p_{\boldsymbol{s} \rightarrow \mathbf{D}}(u, \delta) r_{\mathbf{D} \rightarrow \boldsymbol{s}^{\prime}}(u, \delta) .
$$

Above, $r_{\mathbf{C} \rightarrow \boldsymbol{s}^{\prime}}(u, \delta)$ (resp., $\left.r_{\mathbf{D} \rightarrow \boldsymbol{s}^{\prime}}(u, \delta)\right)$ denotes the probability that, from initial state $\mathbf{C}$ (resp., D), the first excursion from the set $\{\mathbf{C}, \mathbf{D}\}$ is to $\boldsymbol{s}^{\prime}$. Thus the conditional stationary probabilities $\pi_{\boldsymbol{s} \mid \notin\{\mathbf{C}, \mathbf{D}\}}$ are the unique solution to the finite system of equations

$$
\begin{aligned}
\pi_{\boldsymbol{s} \mid \notin\{\mathbf{C}, \mathbf{D}\}}(u, \delta) & =\sum_{\boldsymbol{s}^{\prime} \mid \notin\{\mathbf{C}, \mathbf{D}\}} \pi_{\boldsymbol{s}^{\prime} \mid \notin\{\mathbf{C}, \mathbf{D}\}}(u, \delta) \\
& \times\left(p_{\boldsymbol{s}^{\prime} \rightarrow \boldsymbol{s}}(u, \delta)+p_{\boldsymbol{s}^{\prime} \rightarrow \mathbf{C}}(u, \delta) r_{\mathbf{C} \rightarrow \boldsymbol{s}}(u, \delta)+p_{\boldsymbol{s}^{\prime} \rightarrow \mathbf{D}}(u, \delta) r_{\mathbf{D} \rightarrow \boldsymbol{s}}(u, \delta)\right) .
\end{aligned}
$$

It follows (e.g., from Cramer's rule) that the conditional stationary probabilities $\pi_{\boldsymbol{s} \mid \notin\{\mathbf{C}, \mathbf{D}\}}$ are smooth, rational functions of $(u, \delta) \in(0,1] \times[0, \infty)$.

It only remains to understand how these conditional stationary probabilities behave as $u \rightarrow 0$. We therefore consider the limit of the system $(27)$ as $(u, \delta) \rightarrow\left(0, \delta_{0}\right)$ for some $\delta_{0} \geq 0$. We note that transitions from state $\mathbf{D}$ to a state $\boldsymbol{s} \neq \mathbf{D}$ occur with probability of order $u^{m}$, where $m$ is the number of C's in $s$ (i.e., the number of mutations required for this transition). So as $(u, \delta) \rightarrow\left(0, \delta_{0}\right)$, transitions out of $\mathbf{D}$ become dominated by those going into states with exactly one $\mathrm{C}$. By symmetry, each of these states is equally likely to arise from such a transition. It follows that

$$
\begin{aligned}
& \lim _{(u, \delta) \rightarrow\left(0, \delta_{0}\right)} r_{\mathbf{D} \rightarrow \boldsymbol{s}}(u, \delta) \\
& \quad=\mu_{\boldsymbol{s}}^{\mathrm{C}} \equiv \begin{cases}\frac{1}{N} & \text { if } \exists i \text { such that } s_{i}=\mathrm{C} \text { and } s_{j}=\mathrm{D}, \forall j \neq i, \\
0 & \text { otherwise. }\end{cases}
\end{aligned}
$$


Similarly,

$$
\begin{aligned}
& \lim _{(u, \delta) \rightarrow\left(0, \delta_{0}\right)} r_{\mathrm{C} \rightarrow \boldsymbol{s}}(u, \delta) \\
& =\mu_{s}^{\mathrm{D}} \equiv \begin{cases}\frac{1}{N} & \text { if } \exists i \text { such that } s_{i}=\mathrm{D} \text { and } s_{j}=\mathrm{C}, \forall j \neq i, \\
0 & \text { otherwise. }\end{cases}
\end{aligned}
$$

Let $\gamma(t)$, defined for $t>0$, be a path in $(0,1] \times[0, \infty)$ with $\lim _{t \rightarrow 0} \gamma(t)=\left(0, \delta_{0}\right)$. Substituting $\gamma(t)$ for $(u, \delta)$ in (27) and taking the limit $t \rightarrow 0$, we obtain

$$
\begin{aligned}
\lim _{t \rightarrow 0} \pi_{\boldsymbol{s} \mid \notin\{\mathbf{C}, \mathbf{D}\}}(\gamma(t))= & \sum_{\boldsymbol{s}^{\prime} \mid \notin\{\mathbf{C}, \mathbf{D}\}} \lim _{t \rightarrow 0} \pi_{\boldsymbol{s}^{\prime} \mid \notin\{\mathbf{C}, \mathbf{D}\}}(\gamma(t)) \\
& \times\left(p_{\boldsymbol{s}^{\prime} \rightarrow \boldsymbol{s}}\left(0, \delta_{0}\right)+p_{\boldsymbol{s}^{\prime} \rightarrow \mathbf{C}}\left(0, \delta_{0}\right) \mu_{\boldsymbol{s}}^{\mathrm{D}}+p_{\boldsymbol{s}^{\prime} \rightarrow \mathbf{D}}\left(0, \delta_{0}\right) \mu_{\boldsymbol{s}}^{\mathrm{C}}\right) .
\end{aligned}
$$

Allen and Tarnita [4] (Proof of Theorem 3) show that the system of equations (28) can be solved uniquely for $\left\{\lim _{t \rightarrow 0} \pi_{\boldsymbol{s} \mid \notin\{\mathbf{C}, \mathbf{D}\}}(\gamma(t))\right\}$. Since the coefficients of this system

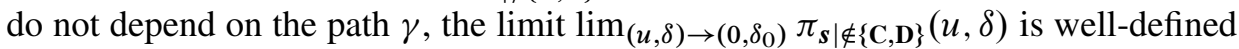
(i.e., independent of the path $\gamma$ ).

Theorem 4. Consider the family of evolutionary Markov chains $\mathcal{M}_{u, \delta}$ with fixed game, graph, and update rule, where the mutation rate $0 \leq u \leq 1$ and selection strength $\delta>0$ are allowed to vary. Then

(1) Strategy $\mathrm{C}$ is favored for fixed $u>0$, in the sense that $\langle x\rangle_{u, \delta}>1 / 2$ for all sufficiently small $\delta>0$, if and only if

$$
\left\langle\frac{\partial\left((1-u) b_{0}-d_{0}\right)}{\partial \delta}\right\rangle_{\substack{\delta=0 \\ s_{0}=\mathrm{C}}}>0 .
$$

(2) Strategy $\mathrm{C}$ is favored in the sense that $\langle x\rangle_{u, \delta}>1 / 2$ for all sufficiently small $u>0$ and $\delta>0$ if and only if

$$
\lim _{u \rightarrow 0}\left\langle\frac{\partial\left(b_{0}-d_{0}\right)}{\partial \delta}\right\rangle_{\substack{\delta=0 \\ s_{0}=\mathrm{C}}}>0 .
$$

(3) Strategy $\mathrm{C}$ is favored for $u=0$, in the sense that $\rho_{\mathrm{C}}>\rho_{\mathrm{D}}$ for all sufficiently small $\delta>0$, if and only if

$$
\lim _{u \rightarrow 0}\left\langle\frac{\partial\left(b_{0}-d_{0}\right)}{\partial \delta}\right\rangle_{\substack{\delta=0 \\ s_{0}=\mathrm{C}}}>0 .
$$

Proof. Claim (1) is proven by Nowak et al. in [67] (Appendix A, Theorem 2). For Claim (2), we observe that the bracketed quantity in the left-hand side of (29) is zero 
for $s \in\{\mathbf{C}, \mathbf{D}\}$; thus the expectation of this quantity is unchanged if it is taken over the conditional stationary distribution $\left\{\pi_{\boldsymbol{s} \mid \notin\{\mathbf{C}, \mathbf{D}\}}\right\}$ instead of the stationary distribution $\left\{\pi_{s}\right\}$. Claim (2) now follows from Lemma 14, i.e., from the fact that the left-hand side of $(29)$ is a rational function of $(u, \delta)$ that is smooth at $(0,0)$ according to Lemma 14. Finally, Claim (3) follows from combining Claim (2) with Theorem 6 of [4].

\section{References}

[1] R. Albert and A.-L. Barabási, Statistical mechanics of complex networks. Rev. Modern Phys. 74 (2002), 47-97. Zbl 1205.82086 MR 2003d:82055

[2] B. Allen, J. Gore, and M. A. Nowak, Spatial dilemmas of diffusible public goods. eLife 2 (2013). doi: 10.7554/eLife.01169

[3] B. Allen and M. A. Nowak, Evolutionary shift dynamics on a cycle. J. Theoret. Biol. 311 (2012), 28-39. MR 2972193

[4] B. Allen and C. E. Tarnita, Measures of success in a class of evolutionary models with fixed population size and structure. J. Math. Biol. 68 (2014), 109-143. Zbl 1280.92045 MR 3147457

[5] B. Allen, A. Traulsen, C. E. Tarnita, and M. A. Nowak, How mutation affects evolutionary games on graphs. J. Theoret. Biol. 299 (2012), 97-105. MR 2899055

[6] T. Antal, H. Ohtsuki, J. Wakeley, P. D. Taylor, and M. A. Nowak, Evolution of cooperation by phenotypic similarity. Proc. Nat. Acad. Sci. U.S.A. 106 (2009), 8597-8600. doi: 10.1073/pnas.0902528106

[7] S. Assenza, J. Gómez-Gardeñes, and V. Latora, Enhancement of cooperation in highly clustered scale-free networks. Phys. Rev. E 78 (2008), 017101. doi: 10.1103/PhysRevE.78.017101

[8] R. Axelrod and W. D. Hamilton, The evolution of cooperation. Science 211 (1981), 1390-1396. Zbl 1225.92037 MR 84f:92030

[9] M. van Baalen and D. A. Rand, The unit of selection in viscous populations and the evolution of altruism. J. Theoret. Biol. 193 (1998), 631-648. doi: 10.1006/jtbi.1998.0730

[10] D. B. Borenstein, Y. Meir, J. W. Shaevitz, and N. S. Wingreen, Non-local interaction via diffusible resource prevents coexistence of cooperators and cheaters in a lattice model. PloS ONE 8 (2013), e63304.

[11] M. Broom, C. Hadjichrysanthou, and J. Rychtár, Evolutionary games on graphs and the speed of the evolutionary process. Proc. Roy. Soc. London Ser. A 466 (2010), 1327-1346. Zbl 1202.91027 MR 2011b:91071

[12] M. Broom and J. Rychtár, Game-theoretical models in biology. Chapman \& Hall/CRC Math. Comput. Biol. Ser., CRC Press, Boca Raton, FL, 2013. Zbl 1264.92002 MR 3052136

[13] M. Cavaliere, S. Sedwards, C. E. Tarnita, M. A. Nowak, and A. Csikász-Nagy, Prosperity is associated with instability in dynamical networks. J. Theoret. Biol. 299 (2012), 126-138. MR 2899059 
[14] Y.-T. Chen, Sharp benefit-to-cost rules for the evolution of cooperation on regular graphs. Ann. Appl. Probab. 23 (2013), 637-664. Zbl 1267.91019 MR 3059271

[15] J. S. Chuang, O. Rivoire, and S. Leibler, Simpson's paradox in a synthetic microbial system. Science 323 (2009), 272-275. doi: 10.1126/science.1166739

[16] J. T. Cox, R. Durrett, and E. A. Perkins, Voter model perturbations and reaction diffusion equations. Astérisque 349 (2013). Zbl 1277.60004 MR 3075759

[17] R. Cressman, The stability concept of evolutionary game theory. Lecture Notes in Biomath. 94, Springer-Verlag, Berlin 1992. Zbl 0763.92006 MR 93j:92020

[18] J. A. Damore and J. Gore, Understanding microbial cooperation. J. Theoret. Biol. 299 (2012), 31-41. MR 2899049

[19] M. S. Datta, K. S. Korolev, I. Cvijovic, C. Dudley, and J. Gore, Range expansion promotes cooperation in an experimental microbial metapopulation. Proc. Nat. Acad. Sci. U.S.A. 110 (2013), 7354-7359. doi: 10.1073/pnas.1217517110

[20] F. Débarre, C. Hauert, and M. Doebeli, Social evolution in structured populations. Nature Communications 5 (2014), 4409.

[21] U. Dieckmann, R. Law, and J. A. J. Metz, The geometry of ecological interactions: simplifying spatial complexity. Cambridge University Press, Cambridge 2000.

[22] R. Durrett and S. Levin, The importance of being discrete (and spatial). Theor. Popul. Biol. 46 (1994), 363-394. Zbl 0846.92027

[23] H. Ebel and S. Bornholdt, Evolutionary games and the emergence of complex networks. Preprint 2002. arXiv:cond-mat/0211666

[24] K. Fehl, D. J. van der Post, and D. Semmann, Co-evolution of behaviour and social network structure promotes human cooperation. Ecol. Lett. 14 (2011), 546-551. doi: 10.1111/j.1461-0248.2011.01615.x

[25] F. Fu, C. Hauert, M. A. Nowak, and L. Wang, Reputation-based partner choice promotes cooperation in social networks. Phys. Rev. E 78 (2008), 026117. doi: 10.1103/PhysRevE.78.026117

[26] C. S. Gokhale and A. Traulsen, Evolutionary games in the multiverse. Proc. Nat. Acad. Sci. U.S.A. 107 (2010), 5500-5504. doi: 10.1073/pnas.0912214107

[27] C. S. Gokhale and A. Traulsen, Strategy abundance in evolutionary many-player games with multiple strategies. J. Theoret. Biol. 283 (2011), 180-191. MR 2973825

[28] J. Gómez-Gardeñes, M. Campillo, L. M. Floría, and Y. Moreno, Dynamical organization of cooperation in complex topologies. Phys. Rev. Lett. 98 (2007), 108103. doi: 10.1103/PhysRevLett.98.108103

[29] J. Gore, H. Youk, and A. van Oudenaarden, Snowdrift game dynamics and facultative cheating in yeast. Nature 459 (2009), 253-256. doi: 10.1038/nature07921

[30] C. Gracia-Lázaro, J. A. Cuesta, A. Sánchez, and Y. Moreno, Human behavior in prisoner's dilemma experiments suppresses network reciprocity. Scientific Reports 2 (2012), Article number: 325. doi: 10.1038/srep00325

[31] C. Gracia-Lázaro, A. Ferrer, G. Ruiz, A. Tarancón, J. A. Cuesta, A. Sánchez, and Y. Moreno, Heterogeneous networks do not promote cooperation when humans play a prisoner's dilemma. Proc. Nat. Acad. Sci. U.S.A. 109 (2012), 12922-12926. doi: 10.1073/pnas.1206681109 
[32] A. Grafen and M. Archetti, Natural selection of altruism in inelastic viscous homogeneous populations. J. Theoret. Biol. 252 (2008), 694-710. MR 2960715

[33] B. L. Granovsky and N. Madras, The noisy voter model. Stochastic Process. Appl. 55 (1995), 23-43. Zbl 0813.60096 MR 95k:60249

[34] J. Grujić, T. Röhl, D. Semmann, M. Milinski, and A. Traulsen, Consistent strategy updating in spatial and non-spatial behavioral experiments does not promote cooperation in social networks. PLoS ONE 7 (2012), e47718. doi: 10.1371/journal.pone.0047718

[35] C. Hadjichrysanthou, M. Broom, and J. Rychtáŕ, Evolutionary games on star graphs under various updating rules. Dyn. Games Appl. 1 (2011), 386-407. Zbl 1252.91016 MR 2012i:91047

[36] C. Hauert and M. Doebeli, Spatial structure often inhibits the evolution of cooperation in the snowdrift game. Nature 428 (2004), 643-646. doi: 10.1038/nature02360

[37] J. Hofbauer and K. Sigmund, Evolutionary games and population dynamics. Cambridge University Press, Cambridge 1998. Zbl 0914.90287 MR 99h:92027

[38] J. Hofbauer and K. Sigmund, Evolutionary game dynamics. Bull. Amer. Math. Soc. (N.S.) 40 (2003), 479-519. Zbl 1049.91025 MR 2004g:91024

[39] L. A. Imhof and M. A. Nowak, Evolutionary game dynamics in a Wright-Fisher process. J. Math. Biol. 52 (2006), 667-681. Zbl 1110.92028 MR 2007f:91017

[40] J. J. Jordan, D. G. Rand, S. Arbesman, J. H. Fowler, and N. A. Christakis, Contagion of cooperation in static and fluid social networks. PLOS ONE 8 (2013), e66199. doi: 10.1371/journal.pone.0066199

[41] T. Julou, T. Mora, L. Guillon, V. Croquette, I. J. Schalk, D. Bensimon, and N. Desprat, Cell-cell contacts confine public goods diffusion inside pseudomonas aeruginosa clonal microcolonies. Proc. Nat. Acad. Sci. U.S.A. 110 (2013), 12577-12582.

[42] B. Kerr, C. Neuhauser, B. J. M. Bohannan, and A. M. Dean, Local migration promotes competitive restraint in a host-pathogen 'tragedy of the commons'. Nature 442 (2006), 75-78. doi: 10.1038/nature04864

[43] T. Killingback and M. Doebeli, Spatial evolutionary game theory: Hawks and Doves revisited. Proc. Roy. Soc. London Ser. B 263 (1996), 1135-1144. doi: 10.1098/rspb.1996.0166

[44] T. Konno, A condition for cooperation in a game on complex networks. J. Theoret. Biol. 269 (2011), 224-233. MR 2974484

[45] R. Kümmerli, A. S. Griffin, S. A. West, A. Buckling, and F. Harrison, Viscous medium promotes cooperation in the pathogenic bacterium Pseudomonas aeruginosa. Proc. Roy. Soc. London Ser. B 276 (2009), 3531-3538. doi: 10.1098/rspb.2009.0861

[46] G. F. Lawler and V. Limic, Random walk: a modern introduction. Cambridge Stud. Adv. Math. 123, Cambridge University Press, Cambridge 2010. Zbl 1210.60002 MR 2012a:60132

[47] M. Le Gac and M. Doebeli, Environmental viscosity does not affect the evolution of cooperation during experimental evolution of colicigenic bacteria. Evolution 64 (2010), 522-533. doi: 10.1111/j.1558-5646.2009.00814.x

[48] E. Lieberman, C. Hauert, and M. A. Nowak, Evolutionary dynamics on graphs. Nature 433 (2005), 312-316. doi: 10.1038/nature03204 
[49] S. Lion and M. van Baalen, Self-structuring in spatial evolutionary ecology. Ecol. Lett. 11 (2008), 277-295. doi: 10.1111/j.1461-0248.2007.01132.x

[50] G. Malécot, Les Mathématiques de l'Hérédité. Masson et Cie., Paris 1948. Zbl 0031.17304 MR 10,314c

[51] H. Matsuda, N. Ogita, A. Sasaki, and K. Sato, Statistical mechanics of population. Progr. Theoret. Phys. 88 (1992), 1035-1049. doi: 10.1143/ptp/88.6.1035

[52] R. M. May and W. J. Leonard, Nonlinear aspects of competition between three species. SIAM J. Appl. Math. 29 (1975), 243-253. Zbl 0314.92008 MR 52 \#12853

[53] J. Maynard Smith, Evolution and the theory of games. Cambridge University Press, Cambridge 1982. Zbl 0526.90102

[54] J. Maynard Smith and G. R. Price, The logic of animal conflict. Nature 246 (1973), 15-18. doi: $10.1038 / 246015 \mathrm{a} 0$

[55] J. Mitteldorf and D. S. Wilson, Population viscosity and the evolution of altruism. $J$. Theoret. Biol. 204 (2000), 481-496. doi: 10.1006/jtbi.2000.2007

[56] E. W. Montroll and G. H. Weiss, Random walks on lattices. II. J. Math. Phys. 6 (1965), 167-181. MR 30 \#2563

[57] M. Nakamaru, H. Matsuda, and Y. Iwasa, The evolution of cooperation in a latticestructured population. J. Theoret. Biol. 184 (1997), 65-81. doi: 10.1006/jtbi.1996.0243

[58] M. E. J. Newman, The structure and function of complex networks. SIAM Rev. 45 (2003), 167-256. Zbl 1029.68010 MR 2005a:05206

[59] M. A. Nowak, Five rules for the evolution of cooperation. Science 314 (2006), 1560-1563. doi: $10.1126 /$ science. 1133755

[60] M. A. Nowak, Evolving cooperation. J. Theoret. Biol. 299 (2012), 1-8. MR 2899045

[61] M. A. Nowak, S. Bonhoeffer, and R. M. May, More spatial games. Internat. J. Bifur. Chaos Appl. Sci. Engrg. 4 (1994), 33-56. Zbl 0884.90147 MR 95a:90213

[62] M. A. Nowak, S. Bonhoeffer, and R. M. May, Spatial games and the maintenance of cooperation. Proc. Nat. Acad. Sci. U.S.A. 91 (1994), 4877-4881. Zbl 0799.92010

[63] M. A. Nowak and R. M. May, Evolutionary games and spatial chaos. Nature 359 (1992), 826-829. doi: 10.1038/359826a0

[64] M. A. Nowak and R. M. May, The spatial dilemmas of evolution. Internat. J. Bifur. Chaos Appl. Sci. Engrg. 3 (1993), 35-78. Zbl 0870.92011 MR 94c:92014

[65] M. A. Nowak, A. Sasaki, C. Taylor, and D. Fudenberg, Emergence of cooperation and evolutionary stability in finite populations. Nature 428 (2004), 646-650. doi: 10.1038 /nature 02414

[66] M. A. Nowak and K. Sigmund, Evolutionary dynamics of biological games. Science $\mathbf{3 0 3}$ (2004), 793-799. doi: 10.1126/science.1093411

[67] M. A. Nowak, C. E. Tarnita, and E. O. Wilson, The evolution of eusociality. Nature 466 (2010), 1057-1062. doi: 10.1038/nature09205

[68] H. Ohtsuki, C. Hauert, E. Lieberman, and M. A. Nowak, A simple rule for the evolution of cooperation on graphs and social networks. Nature 441 (2006), 502-505. doi: $10.1038 /$ nature04605 
[69] H. Ohtsuki and M. A. Nowak, Evolutionary games on cycles. Proc. Roy. Soc. London Ser. B 273 (2006), 2249-2256. doi: 10.1098/rspb.2006.3576

[70] H. Ohtsuki and M. A. Nowak, The replicator equation on graphs. J. Theoret. Biol. 243 (2006), 86-97. MR 2279323

[71] H. Ohtsuki and M. A. Nowak, Evolutionary stability on graphs. J. Theoret. Biol. 251 (2008), 698-707. MR 2949113

[72] H. Ohtsuki, M. A. Nowak, and J. M. Pacheco, Breaking the symmetry between interaction and replacement in evolutionary dynamics on graphs. Phys. Rev. Lett. 98 (2007), 108106. doi: 10.1103/PhysRevLett.98.108106

[73] H. Ohtsuki, J. M. Pacheco, and M. A. Nowak, Evolutionary graph theory: breaking the symmetry between interaction and replacement. J. Theoret. Biol. 246 (2007), 681-694. MR 2008b:92081

[74] J. M. Pacheco, A. Traulsen, and M. A. Nowak, Active linking in evolutionary games. $J$. Theoret. Biol. 243 (2006), 437-443. MR 2280046

[75] J. M. Pacheco, A. Traulsen, and M. A. Nowak, Coevolution of strategy and structure in complex networks with dynamical linking. Phys. Rev. Lett. 97 (2006), 258103. doi: 10.1103/PhysRevLett.97.258103

[76] M. Perc, J. Gómez-Gardeñes, A. Szolnoki, L. M. Floría, and Y. Moreno, Evolutionary dynamics of group interactions on structured populations: a review. J. R. Soc. Interface 10 (2013), 20120997. doi: 10.1098/rsif.2012.0997

[77] M. Perc and A. Szolnoki, Coevolutionary games-A mini review. BioSystems 99 (2010), 109-125. doi: 10.1016/j.biosystems.2009.10.003

[78] F. L. Pinheiro, F. C. Santos, and J. M. Pacheco, How selection pressure changes the nature of social dilemmas in structured populations. New J. Phys. 14 (2012), 073035. doi: 10.1088/1367-2630/14/7/073035

[79] D. G. Rand, S. Arbesman, and N. A. Christakis, Dynamic social networks promote cooperation in experiments with humans. Proc. Nat. Acad. Sci. U.S.A. 108(2011), 19193-19198. doi: 10.1073/pnas.1108243108

[80] D. G. Rand and M. A. Nowak, Human cooperation. Trends in cognitive sciences 17 (2013), 413-425. doi: 10.1016/j.tics.2013.06.003

[81] C. P. Roca, J. A. Cuesta, and A. Sánchez, Effect of spatial structure on the evolution of cooperation. Phys. Rev. E 80 (2009), 046106. doi: 10.1103/PhysRevE.80.046106

[82] C. P. Roca, J. A. Cuesta, and A. Sánchez, Evolutionary game theory: Temporal and spatial effects beyond replicator dynamics. Phys. Life Rev. 6 (2009), 208-249. doi: 10.1016/j.plrev.2009.08.001

[83] F. Rousset and S. Billiard, A theoretical basis for measures of kin selection in subdivided populations: finite populations and localized dispersal. J. Evol. Biol. 13 (2000), 814-825. doi: 10.1046/j.1420-9101.2000.00219.x

[84] F. C. Santos and J. M. Pacheco, Scale-free networks provide a unifying framework for the emergence of cooperation. Phys. Rev. Lett. 95 (2005), 98104. doi: 10.1103/PhysRevLett.95.098104

[85] F. C. Santos, M. D. Santos, and J. M. Pacheco, Social diversity promotes the emergence of cooperation in public goods games. Nature 454 (2008), 213-216. doi: 10.1038/nature06940 
[86] K. Sato, N. Konno, and T. Yamaguchi, Paper-scissors-stone game on trees. Mem. Muroran Inst. Technol. (1997), 109-114. MR 1609551

[87] P. Shakarian, P. Roos, and A. Johnson, A review of evolutionary graph theory with applications to game theory. Biosystems 107 (2012), 66-80.

doi: 10.1016/j.biosystems.2011.09.006

[88] E. H. Simpson, Measurement of diversity. Nature 163 (1949), 688. doi: $10.1038 / 163688 \mathrm{a} 0$

[89] B. Skyrms, Evolution of the social contract. Cambridge University Press, Cambridge 1996.

[90] B. Skyrms and R. Pemantle, A dynamic model of social network formation. Proc. Nat. Acad. Sci. U.S.A. 97 (2000), 9340-9346. doi: 10.1073/pnas.97.16.9340

[91] M. Slatkin, Inbreeding coefficients and coalescence times. Genet. Res. 58 (1991), 167-175. doi: 10.1017/S0016672300029827

[92] B. C. Stacey, A. Gros, and Y. Bar-Yam, Eco-evolutionary feedback in host-pathogen spatial ecology. Preprint 2011. arXiv:1110.3845

[93] S. H. Strogatz, Exploring complex networks. Nature 410 (2001), 268-276. doi: $10.1038 / 35065725$

[94] S. Suri and D. J. Watts, Cooperation and contagion in web-based, networked public goods experiments. PLoS ONE 6 (2011), e16836. doi: 10.1371/journal.pone.0016836

[95] G. Szabó and G. Fáth, Evolutionary games on graphs. Phys. Rep. 446 (2007), 97-216. MR 2008e:91013

[96] G. Szabó and C. Hauert, Phase transitions and volunteering in spatial public goods games. Phys. Rev. Lett. 89 (2002), 118101. doi: 10.1103/PhysRevLett.89.118101

[97] G. Szabó, A. Szolnoki, and R. Izsák, Rock-scissors-paper game on regular small-world networks. J. Phys. A 37 (2004), 2599-2609. Zbl 1055.82021 MR 2005a:82104

[98] A. Szolnoki and M. Perc, Resolving social dilemmas on evolving random networks. Europhys. Lett. 86 (2009), 30007. doi: 10.1209/0295-5075/86/30007

[99] A. Szolnoki, M. Perc, and Z. Danku, Making new connections towards cooperation in the prisoner's dilemma game. Europhys. Lett. 84 (2008), 50007. doi: 10.1209/0295-5075/84/50007

[100] A. Szolnoki, M. Perc, and G. Szabó, Phase diagrams for three-strategy evolutionary prisoner's dilemma games on regular graphs. Phys. Rev. E 80 (2009), 056104. doi: 10.1103/PhysRevE.80.056104

[101] A. Szolnoki and G. Szabó, Phase transitions for rock-scissors-paper game on different networks. Phys. Rev. E 70 (2004), 037102. doi: 10.1103/PhysRevE.70.037102

[102] K.-i. Tainaka, Lattice model for the Lotka-Volterra system. J. Phys. Soc. Jpn. 57 (1988), 2588-2590. doi: 10.1143/JPSJ.57.2588

[103] K.-i. Tainaka, Stationary pattern of vortices or strings in biological systems: Lattice version of the Lotka-Volterra model. Phys. Rev. Lett. 63 (1989), 2688. doi: 10.1103/PhysRevLett.63.2688

[104] K.-i. Tainaka, Vortices and strings in a model ecosystem. Phys. Rev. E 50 (1994), 3401. doi: 10.1103/PhysRevE.50.3401 
[105] C. E. Tarnita, T. Antal, H. Ohtsuki, and M. A. Nowak, Evolutionary dynamics in set structured populations. Proc. Nat. Acad. Sci. U.S.A. 106 (2009), 8601-8604. doi: 10.1073/pnas.0903019106

[106] C. E. Tarnita, H. Ohtsuki, T. Antal, F. Fu, and M. A. Nowak, Strategy selection in structured populations. J. Theoret. Biol. 259 (2009), 570-581. MR 2973175

[107] C. E. Tarnita, N. Wage, and M. A. Nowak, Multiple strategies in structured populations. Proc. Nat. Acad. Sci. U.S.A. 108 (2011), 2334-2337. doi: 10.1073/pnas.1016008108

[108] C. Taylor, D. Fudenberg, A. Sasaki, and M. A. Nowak, Evolutionary game dynamics in finite populations. Bull. Math. Biol. 66 (2004), 1621-1644. MR 2008e:91014

[109] P. Taylor, T. Lillicrap, and D. Cownden, Inclusive fitness analysis on mathematical groups. Evolution 65 (2011), 849-859. doi: 10.1111/j.1558-5646.2010.01162.x

[110] P. D. Taylor, T. Day, and G. Wild, Evolution of cooperation in a finite homogeneous graph. Nature 447 (2007), 469-472. doi: 10.1038/nature05784

[111] P. D. Taylor and L. B. Jonker, Evolutionarily stable strategies and game dynamics. Math. Biosci. 40 (1978), 145-156. Zbl 0395.90118 MR 58 \#9351

[112] A. Traulsen, C. Hauert, H. De Silva, M. A. Nowak, and K. Sigmund, Exploration dynamics in evolutionary games. Proc. Natl. Acad. Sci. U.S.A. 106 (2009), 709-712. Zbl 1202.91029 doi: 10.1073/pnas.0808450106

[113] A. Traulsen, M. A. Nowak, and J. M. Pacheco, Stochastic dynamics of invasion and fixation. Phys. Rev. E 74 (2006), 011909. doi: 10.1103/PhysRevE.74.011909

[114] A. Traulsen, D. Semmann, R. D. Sommerfeld, H.-J. Krambeck, and M. Milinski, Human strategy updating in evolutionary games. Proc. Natl. Acad. Sci. U.S.A. 107 (2010), 2962-2966. doi: 10.1073/pnas.0912515107

[115] M. van Veelen and M. A. Nowak, Multi-player games on the cycle. J. Theoret. Biol. 292 (2012), 116-128. MR 2878667

[116] J. Y. Wakano, H. Ohtsuki, and Y. Kobayashi, A mathematical description of the inclusive fitness theory. Theor. Popul. Biol. 84 (2013), 46-55. Zbl 1275.92082 doi: 10.1016/j.tpb.2012.11.007

[117] J. Wang, S. Suri, and D. J. Watts, Cooperation and assortativity with dynamic partner updating. Proc. Natl. Acad. Sci. U.S.A. 109 (2012), 14363-14368. doi: 10.1073/pnas.1120867109

[118] J. W. Weibull, Evolutionary game theory. MIT Press, Cambridge, MA, 1995. Zbl 0879.90206 MR 96f:90004

[119] B. Wu, D. Zhou, F. Fu, Q. Luo, L. Wang, and A. Traulsen, Evolution of cooperation on stochastic dynamical networks. PLoS ONE 5 (2010), e11187. doi: 10.1371/journal.pone.0011187

[120] H.-X. Yang, Z.-X. Wu, and W.-B. Du, Evolutionary games on scale-free networks with tunable degree distribution. Europhys. Lett. 99 (2012), 10006. doi: 10.1209/0295-5075/99/10006

Received February 5, 2014; revised February 24, 2014 